\title{
An Anisotropic Elasto-Plastic Model Based on an Isotropic Formulation
}

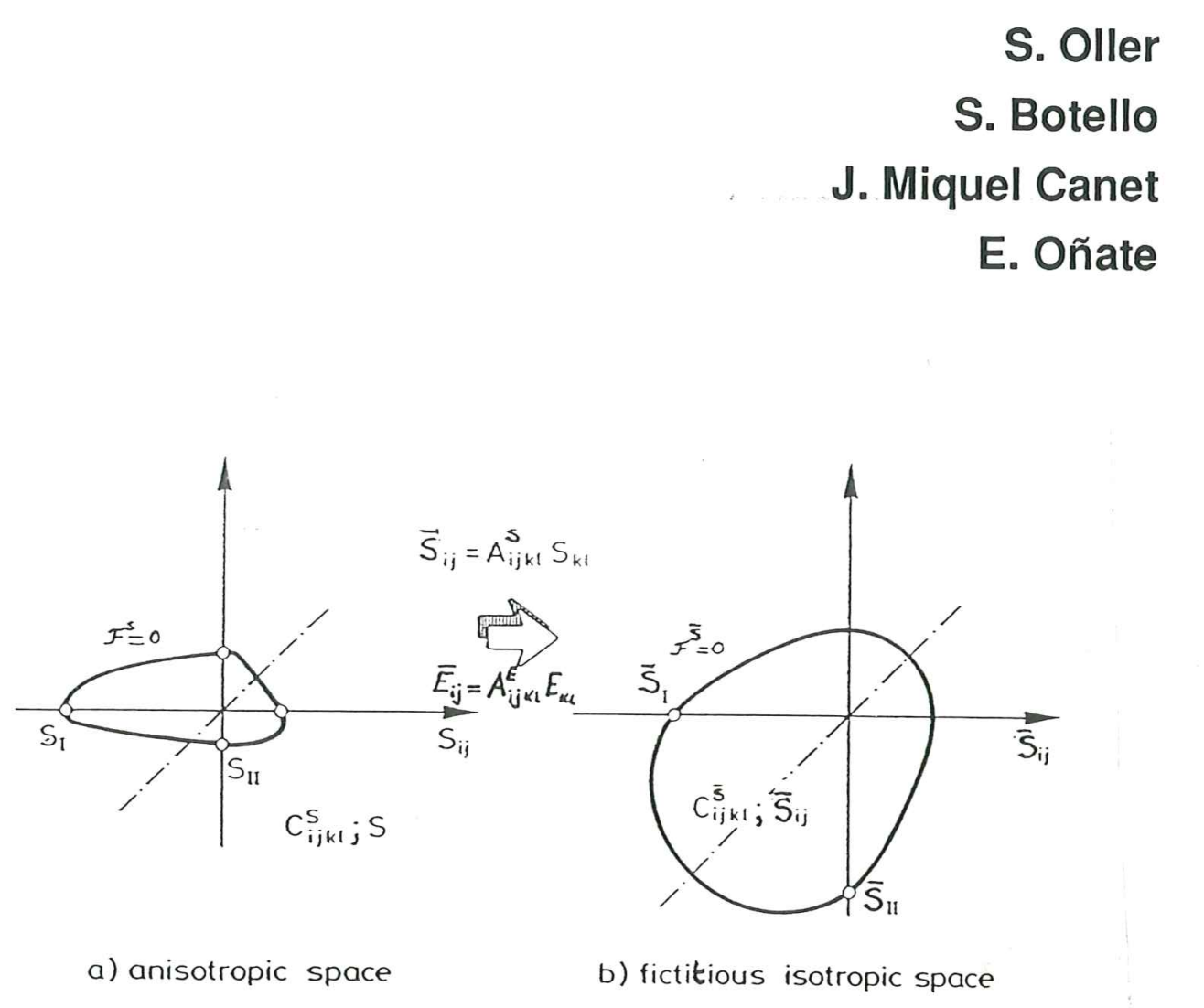




\section{An Anisotropic Elasto-Plastic Modell B

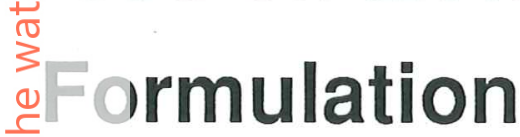

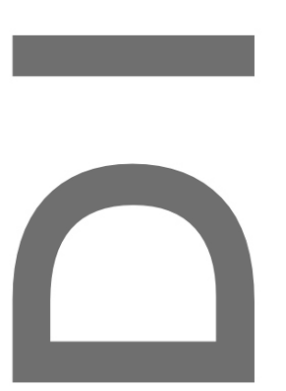

S. Oller

S. Botello

J. Miquel Canet

E. Oñate

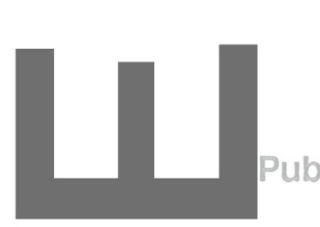

Publioation CIMNE № 41, November 1993

$+$

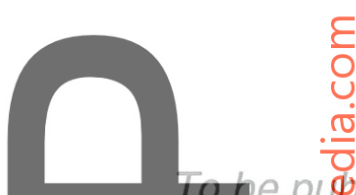

To be puglished in Engineering Computations

âtitán s/n, 08034 Barcelona, España

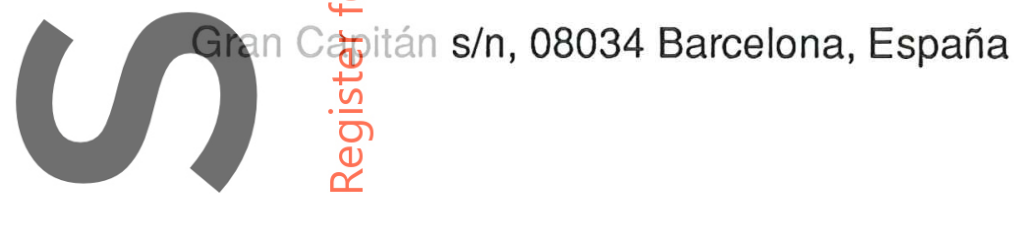




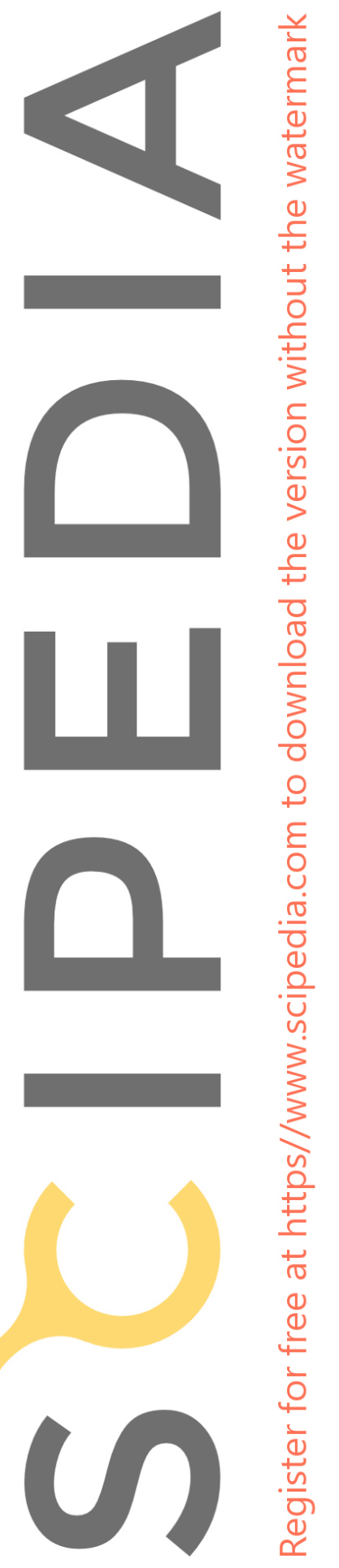




\title{
AN ANISOTROPIC ELASTO-PLASTIC MODEL BASED ON AN ISOTROPIC FORMULATION
}

\author{
S. Oller, S. Botello, J. Miquel Canet and E. Oñate
}

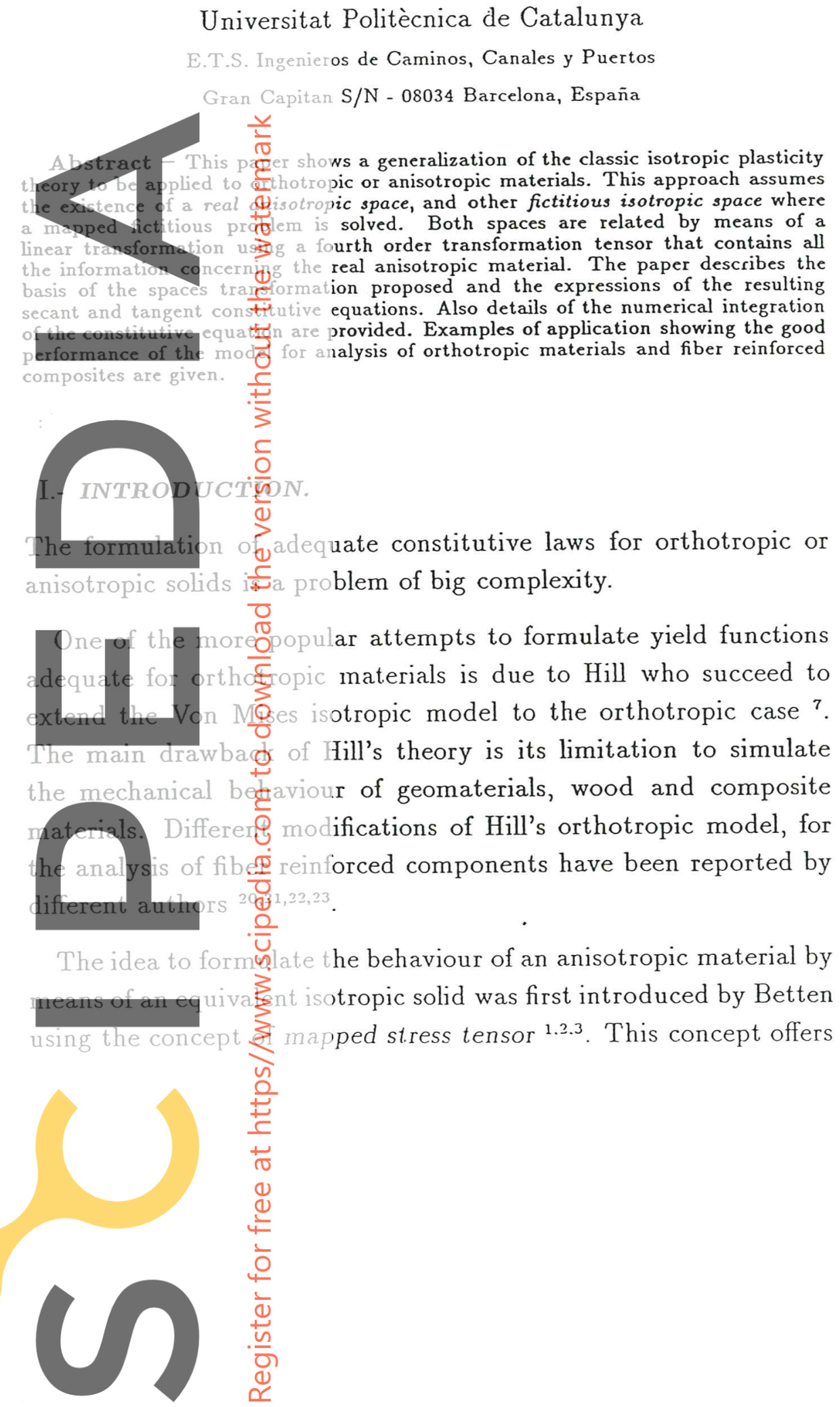


the possibility of using all the advantages of the well known isotropic models; consequently it has many computational advantages.

The authors have developed a generalization of standard isotropic plasticity theory for the analysis of anisotropic solids in previous works 18,25,26. The basic idea was to model the behaviour of an the anisotropic solid by means of a fictitious isotropic solid. A basic assumption of the model was that the elastic strain is unique for both the real and fictitious spaces. This situation introduces a limitation in thenanisotropic mapped theory, because it involves a proportionality and the elasticity modulus foech material direction $\left(f_{11}^{s} / E_{11}=\right.$ $\left.f_{22}^{S} / E_{22}=\cdots=f_{33}^{S} / E_{23}\right)$. In $\frac{\pi}{3}$ he present work a generalization of such basic theory is introduced.

The anisowopic behaviour ifformulated by means of the fictitious isotropic stress and strain tegsors which result from the tensor transformations of the real ss sesses and strains. This allows to use the sam yield and poteffial functions derived for standard isotropic materials, wheas $\frac{0}{\frac{0}{9}}$ ll the relevant information on the material anisotropy properties transformations tensors anly. Qhe material parameters involved in these tensors can be defined fron adequate experimental tests. This model is here terned "iso ropiomapped model for non-proportional materials".

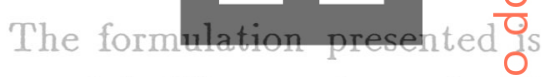

completely general and it allows to model different class of orthotropic and anisotropic materials typical in composites. The mog del seems to be particularly suited to be applied for malysis of ơmultiphase materials such as fiber reinforced conposites and coragrete.

The layout of the paper isuthe following. In next section the properties of the constitutive for a general anisotropic material are described together with the stress and 
strain transformations and the derivation of the secant and tangent constitutive relationships.

Details of the integration of the constitutive equation are given next. The implementation aspects into a finite element code are detailed in a next section.

Finally, the model is applied to three particular problems: 1.The study of the fibers orientation in a fiber reinforced composite. 2.- The comparison of the results in the analysis of an orthotropic material with thosevprovided by standard Von-Mises-Hill ${ }^{7}$ theory,

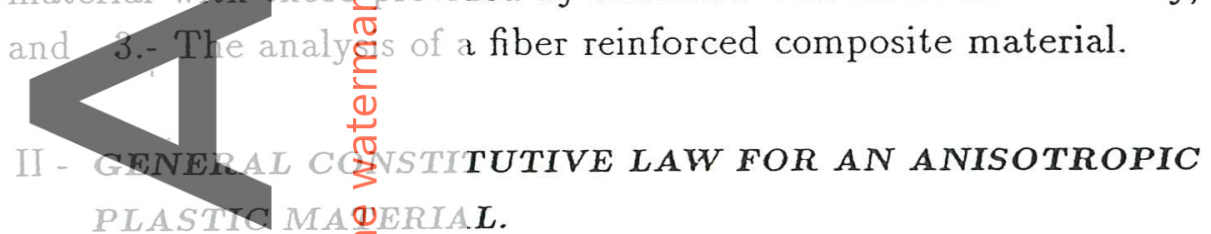
PLASTIC MADERIAL.

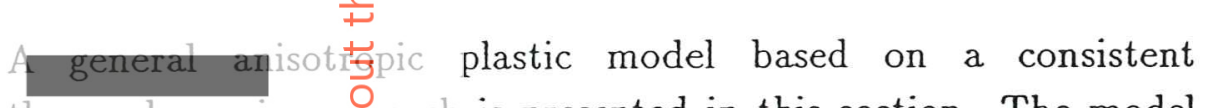
thermodynamic ap O

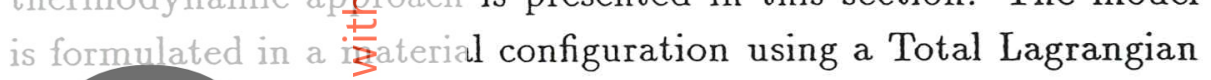
Kinepatios 12. Tife model, as presented here, can deal with non
linear problems iniwhling large plastic strains and small elastic
strains 9,12,17. Obviosly, simpler orthotropic and isotropic plasticity
models are readily obtained as particular cases of the model
proposed. proposed.
Il.1 - Yield and
It wil be ass
functions are defir

tential functions. Space transformation tensor. that both yield and plastic potential the Piola-Kirchoff stress space (material configuration), as:

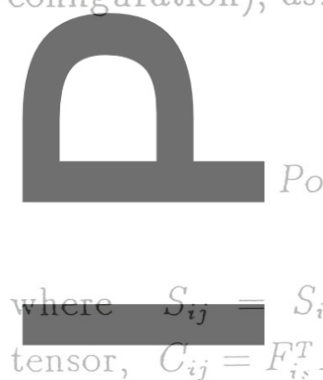

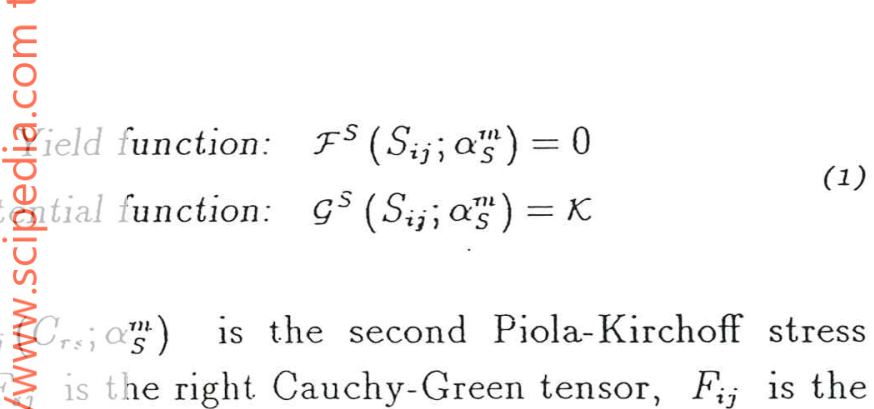


deformation gradient, $\alpha_{S}^{m}$ is a set of $m$ internal plastic variables, and $\mathcal{K}$ is a constant parameter.

The yield and plastic potential functions are isotropic if the invariance condition ${ }^{1,3,14}$

$$
\begin{aligned}
& \mathcal{F}^{S}\left(a_{i p} a_{j q} S_{p q} ; \alpha_{S}^{n_{2}}\right)=\mathcal{F}^{S}\left(S_{i j} ; \alpha_{S}^{m 2}\right)=0 \\
& \mathcal{G}^{S}\left(a_{i p} a_{j q} S_{1 q} ; \alpha_{S}^{m 2}\right)=\mathcal{G}^{S}\left(S_{i j} ; \alpha_{S}^{m^{2}}\right)=\mathcal{K}
\end{aligned}
$$

is satisfied for any orthogon $\frac{\text { at }}{\text { at }}$ transformation ( $a_{i k} a_{j k}=\delta_{i j}$, where $a_{i j}$ is a unit diagonal tens $\frac{E}{2}$, and $\delta_{i j}$ is the Kronecker tensor). Obviously, isotropic materiales satisfy the invariance condition. This can be simply checked by withing eqs.(2) in terms of the firsi three stress invariant, $I_{1}, I_{2}$ and $\underset{f}{I}\left(\right.$ see ref. $\left.{ }^{14}\right)$, i.e:

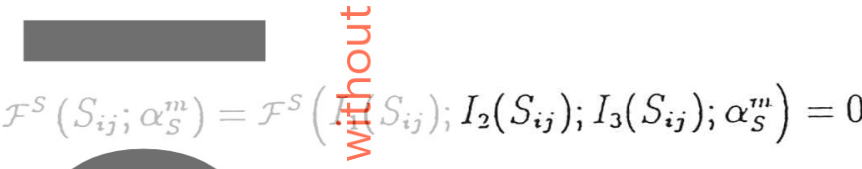

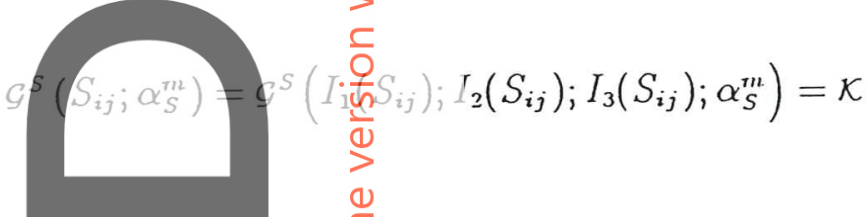

For materials satisfying pertic incompressibility eq.(3) can be written in terms of the invariont of the deviatoric stress tensor as:

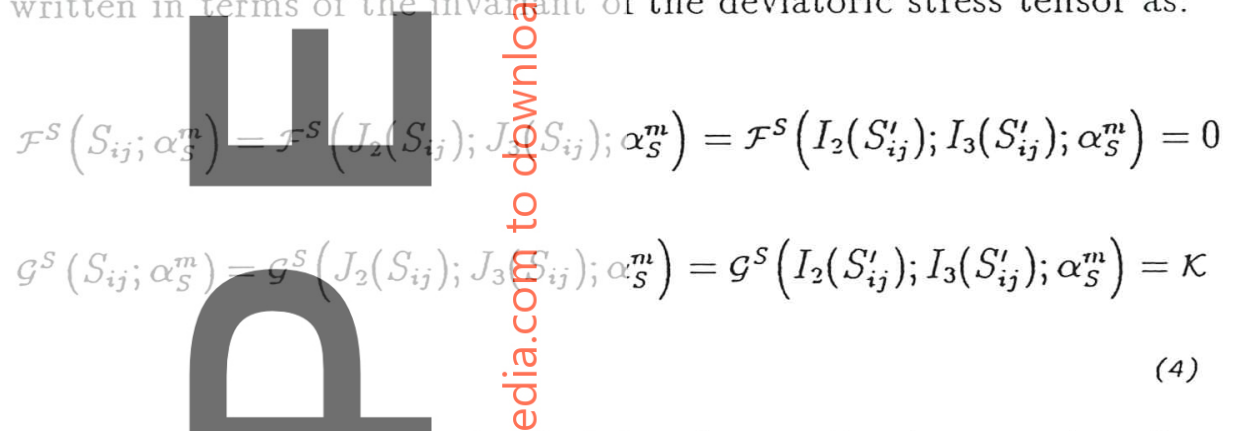

Traditional procedures fog $\frac{\text { G }}{\mathrm{U}}$ eriving the constitutive equations for anisotropic elasto-plastic materials are based on the description of appropriate yield and 3 otential functions in terms of the characteristic material presties 21.22. Satisfaction of the

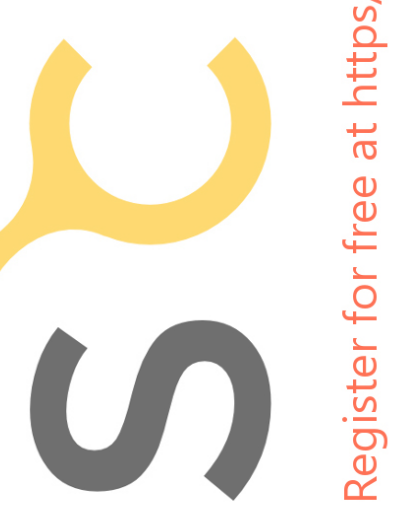


invariance condition in these cases is difficult and not always possible. A procedure to guarantee this condition proposed in this work is to define the properties of the real anisotropic solid in terms of those for a fictitious isotropic solid ${ }^{1.2}$. This is achieved by relating the stresses in the real and fictitious spaces using the following linear transformation (see Figure 1):

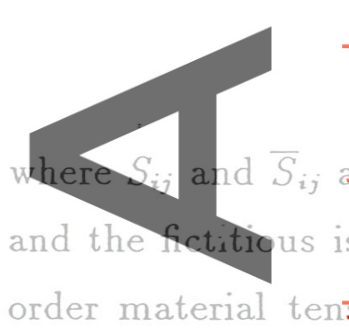

$$
\text { 는 } \quad \bar{S}_{i j}=A_{i j k l}^{S} S_{k l l}
$$

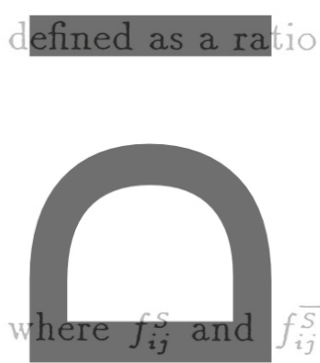

the stress tensor in the real anisotropic solid Botropic solid, respectively and $A_{i j k l}^{S}$ is a fourth. $\frac{(1)}{50}$

, termed stress space transformation tensor ōthe naterial strength, i.e.: the real and fictitions solids, respectively.

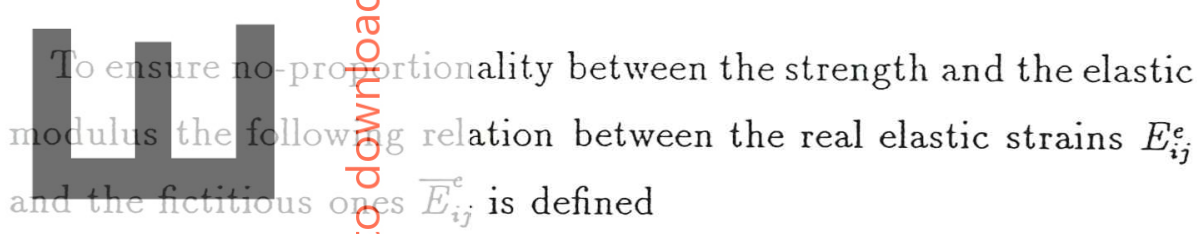

$$
A_{i j k l}^{S}=f_{i k}^{\bar{S}} f_{j l}^{S-1}
$$

the yield strength tensors corresponding to
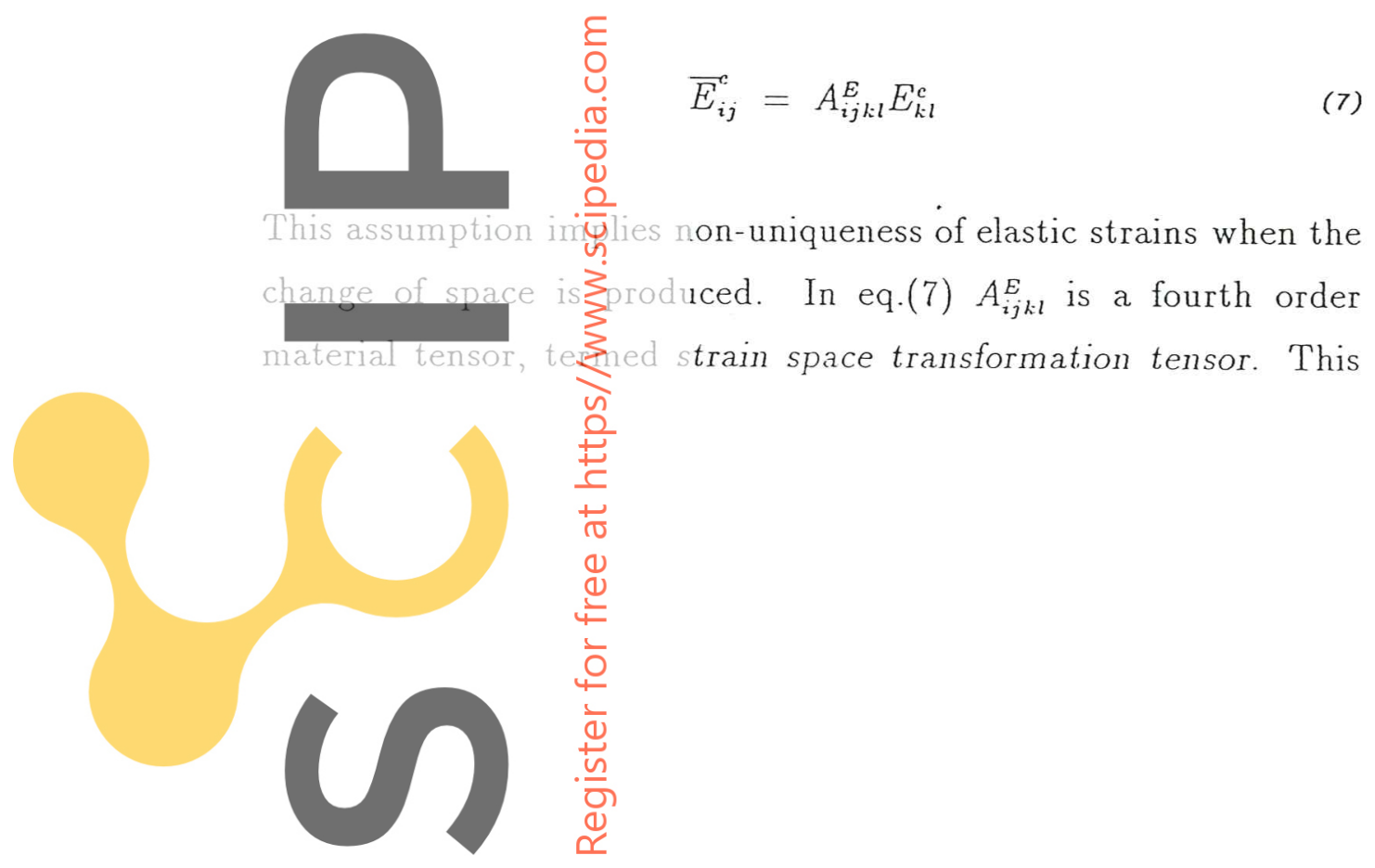

lies non-uniqueness of elastic strains when the Kroduced. In eq.(7) $A_{i j k l}^{E}$ is a fourth order ed strain space transformation tensor. This 
can be derived from equation (5) as follows:

$$
\begin{aligned}
& \bar{S}_{i k} S_{j l}^{-1}=A_{i j k l}^{S}=\left(c^{\bar{S}_{i k r s}} \bar{E}_{\tau s}^{c}\right)\left(c^{S}{ }_{j l m l n} E_{m+n}^{e}\right)^{-1} \\
& =\mathcal{C}^{\bar{S}}{ }_{i k r s} \underbrace{\overline{E_{r s}^{c} E_{m n}^{c}{ }^{-1}}}_{A_{r \leqslant m n}^{E}} C^{S}{ }_{m n j l}^{-1} \\
& \text { and hence. }
\end{aligned}
$$

Eq. (8b) allows to derive the trelationship between the constitutive tensors in the real $\mathcal{C}^{s}{ }_{i j k l}$ and $\vec{C}_{\text {fictitious }} \overline{\mathcal{C}}{ }_{i j k l}$ spaces. This is:

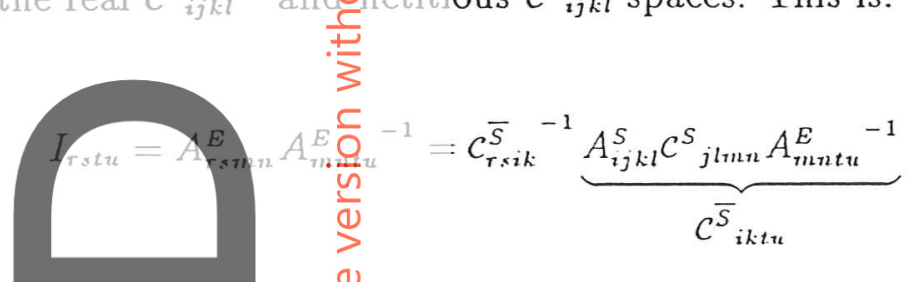

or the inverse relation. $\frac{Y}{+}$

$\stackrel{(1)}{\rightleftarrows}$

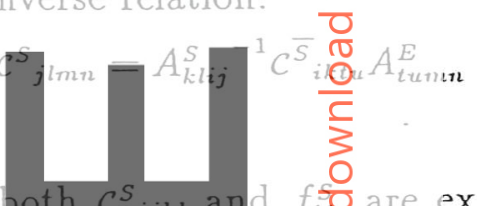

Note that both $\mathcal{C}^{S}{ }_{i j k l}$ and $f_{i j}$ are expressed in a global reference system. This mean that pHor to the derivation of $A_{i j k l}^{S}$ and $A_{i j k l}^{E}$ the following transform gुtions to the global reference system are require

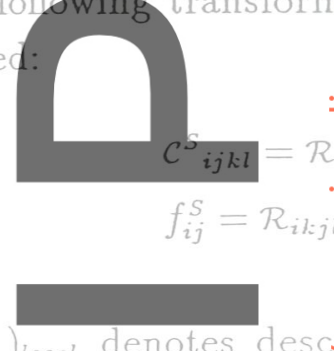

where () local denotes descution in a local coordinate system.

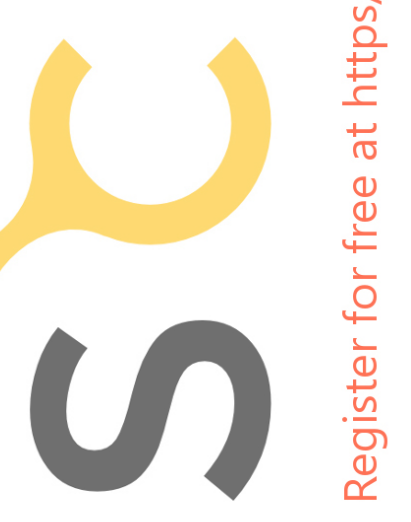


The rotation tensor* $\mathcal{R}_{i j k l}$ takes into account the angles between the local principal directions of the anisotropic material and those of the global coordinate system. Superindexes $S$ and $\bar{S}$ denote hereafter variables in the real (anisotropic) and fictitious (isotropic) spaces, respectively.

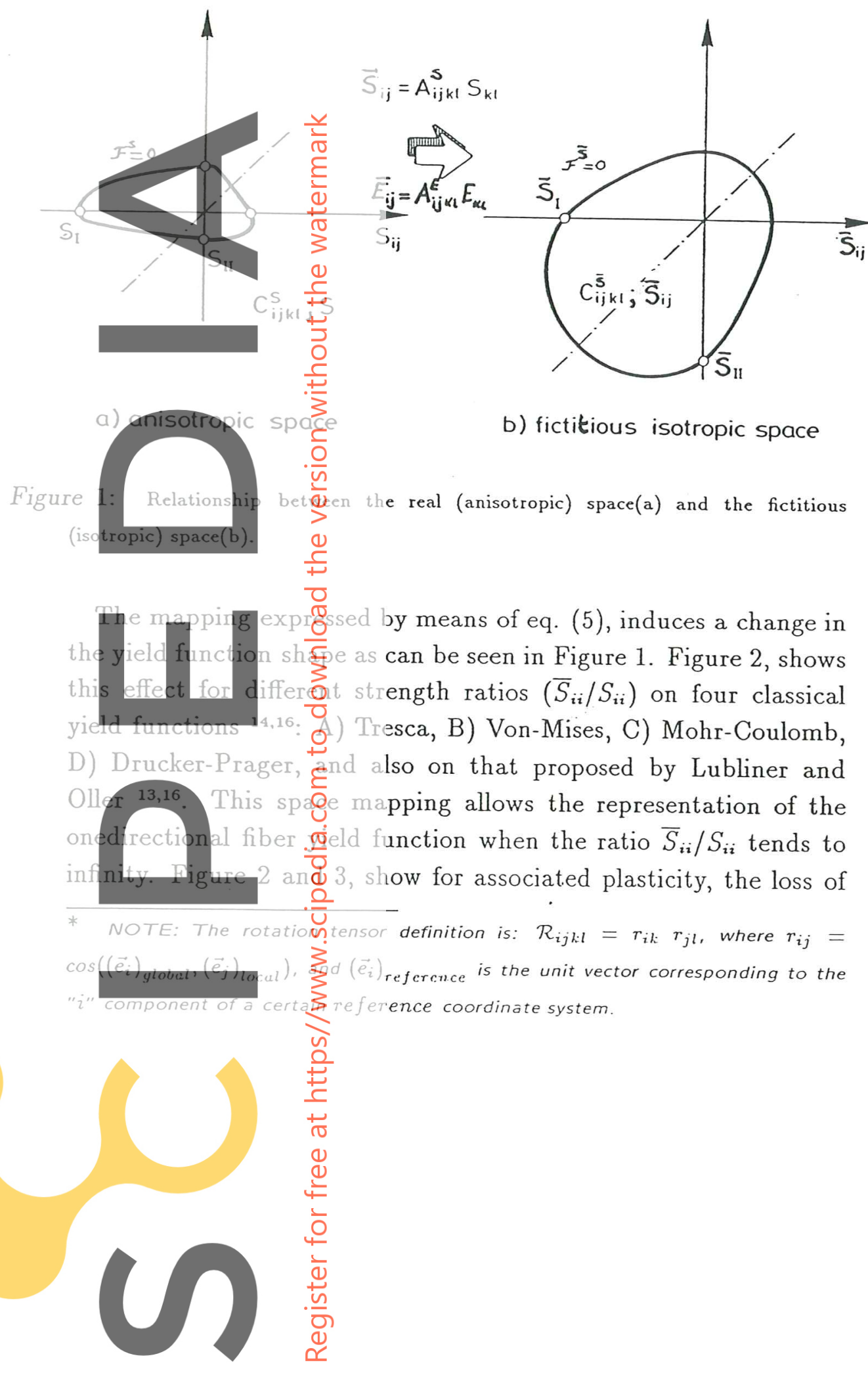


the strength in a given direction while in the normal one there is a plastic flow growth in the same proportion.

Assuming that all the information concerning material anisotropy is contained in the tensor $A_{i j k l}^{S}$, the yield and plastic potential functions for the anisotropic solid are defined as:

$$
\begin{aligned}
& \mathcal{F}^{S}\left(S_{i j} ; \alpha_{S}^{m n}\right)=\mathcal{F}^{\bar{S}}\left(S_{i j} ; A_{i j k l}^{S} ; \alpha \frac{m_{L}}{S}\right)=\mathcal{F}^{\bar{S}}\left(\bar{S}_{i j} ; \alpha \frac{m}{S}\right)=0 \\
& \mathcal{G}^{S}\left(S_{i j} ; \alpha_{S}^{m}\right)=\mathcal{G}^{\bar{S}}\left(S_{i j} ; A_{i j k l}^{S} ; \alpha \frac{m}{S}\right)=\mathcal{G}^{\bar{S}}\left(\bar{S}_{i j} ; \alpha \frac{m}{S}\right)=\mathcal{K}
\end{aligned}
$$

It is therefore concluded that the yield and plastic potential functions for any anisotropic solid can be simple defined in terms of an irreducible basis of the invariants of tensor $\bar{S}_{i j}$. Usually, a finite number of stress invariants are involved in the definition of the yield and potential functions (i.e.: $I_{1}, J_{2}$ and sometimes $J_{3}$ ). These invariants are elements of the system of invariants which are only considered. Therefore the formulation presented is a simplified theory but very useful to solve practical problems ${ }^{1}$.

In the following sections the main relationships characterizing an anisotropic elasto-plastic solid formulated as an ideal isotropic solid using the spaces transformation expressed by eqs.(5) and (7) are derived.

\section{II.2 .- Secant constitutive equation.}

The constitutive equation for an anisotropic material is obtained by writing the dissipation occurring in an isothermic elastoplastic process in the real anisotropic space. From the first Classius-Plank condition $10,11,12,14,17,18$ the following expression for the mechanical dissipation power is obtained:

$$
\Xi_{m e c .}^{S}=\left(\frac{S_{i j}}{m^{0}}-\frac{\partial \Psi^{S}}{\partial E_{i j}^{e}}\right) \dot{E}_{i j}^{e} \div \frac{S_{i j} \dot{E}_{i j}^{\prime \prime}}{m^{0}}-\sum_{m} \frac{\partial \Psi^{S}}{\partial \alpha_{S}^{m h}} \dot{\alpha}_{S}^{m n} \geq 0
$$


(A)

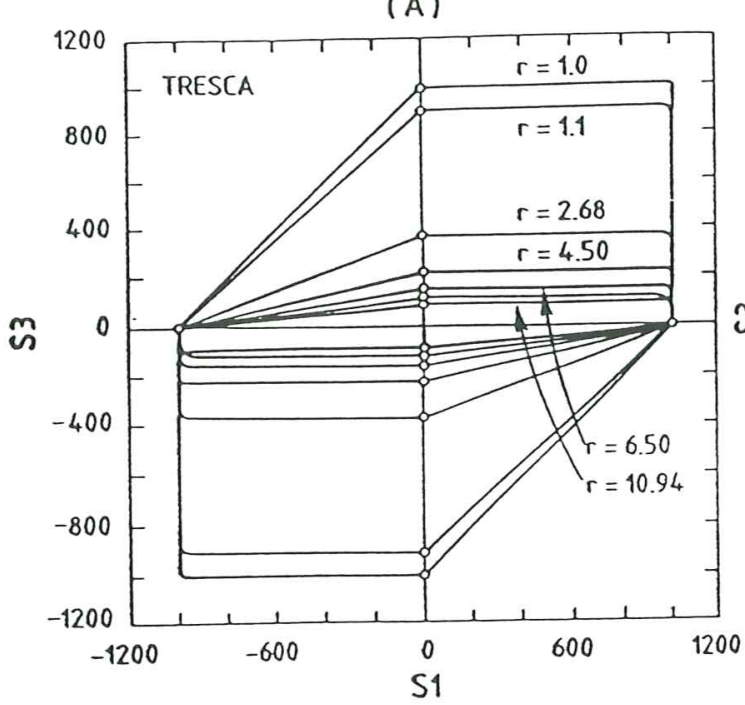

(C)

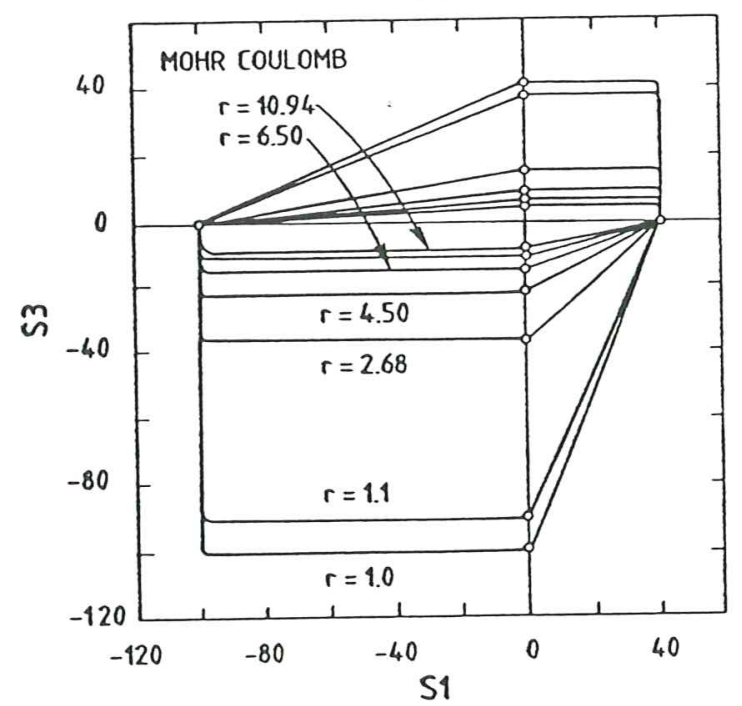

(B)

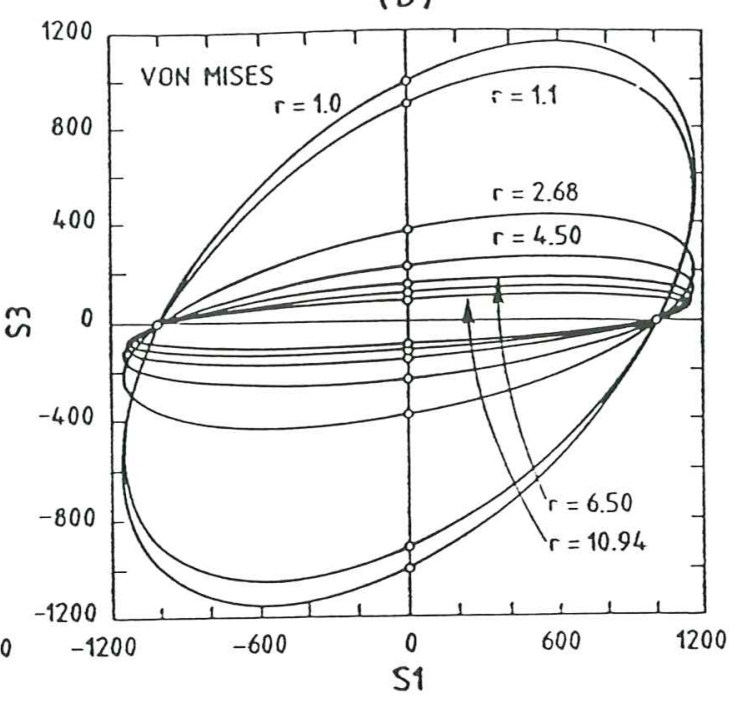

(D)

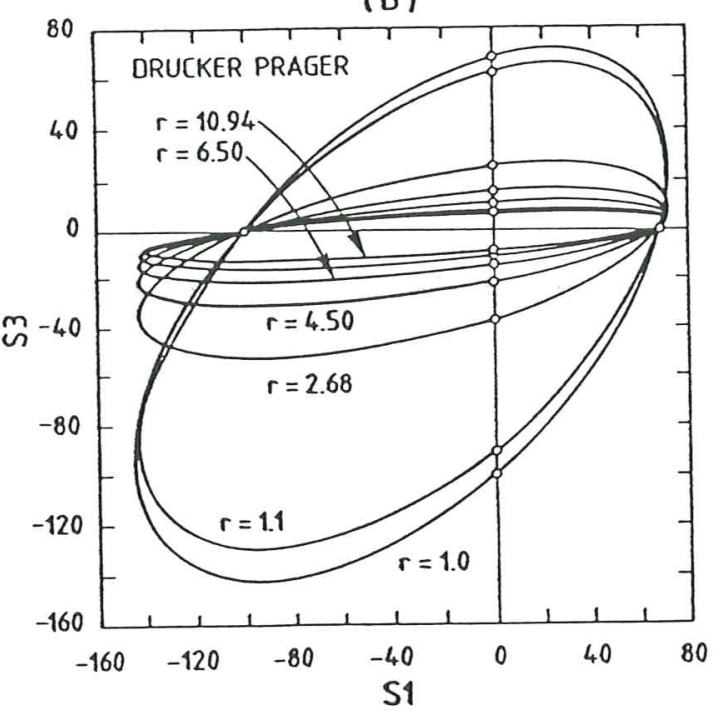

(E)

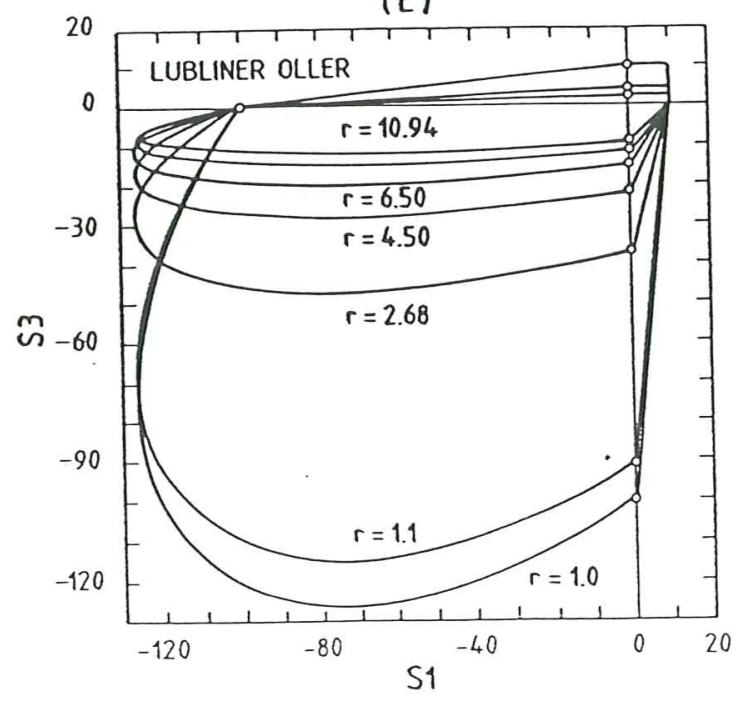

Figure 2: Shape effect for different strength ratios $\left(\bar{S}_{i i} / S_{i:}\right)$ on several yiele functions: A) Tresca, B) Von-Mises, C) Mohr-Coulomb, Di Drucker-Prager, and E: LublinerOlier. 
Applying Colleman's method 11 to guarantee the condition of positive dissipation in eq. (11), the secant constitutive equation is obtained as:

$$
S_{i j}=m^{0} \frac{\partial \Phi^{S}\left(E_{i j}^{c} ; \alpha_{S}^{n n}\right)}{\partial E_{i j}^{c}}
$$

where $\Psi^{S}$ is the free energy of material formulated in the material configuration under real stress state and $m^{0}$ the density in the material configuration. The free energy is assumed to be of the form:

$$
\begin{aligned}
\Psi^{S}\left(E_{i j}^{e} ; \alpha_{S}^{m}\right)= & \Psi^{S^{e}}\left(E_{i j}^{e}\right)+\Psi^{S^{p}}\left(\alpha_{S}^{m_{2}}\right)= \\
& \left(\frac{1}{2 m_{o}} E_{i j}^{e} \mathcal{C}^{S}{ }_{i j k l} E_{k l}^{e}\right)+\Psi^{S{ }^{\prime}}\left(\alpha_{S}^{m}\right)
\end{aligned}
$$

where $\Psi^{S^{e}}$ and $\Psi^{S^{\prime}}$ denote the elastic and plastic free energy contributions, and the free variable $E_{i j}^{c}$ is the fictitious elastic strain in the material configuration as proposed by Green-Naghdi 6,11,12 and defined as:

$$
E_{i j}^{e}=E_{i j}-E_{i j}^{p^{\prime}}=\frac{1}{2}\left(C_{i j}-I_{i j}\right)-\int_{0}^{t} \dot{E}_{i j}^{y^{\prime}} d t
$$

where $E_{i j}^{p}$ is the Lagrangian plastic strain, $C_{i j}$ is the right CauchyGreen tensor and $\dot{E}_{i j}^{p}$ the plastic strain rate defined in the material configuration as explained in a next section.

Substitution of eq.(9) into eq.(13) allows to rewrite the free energy in terms of the constitutive tensor $\mathcal{C}^{\bar{S}_{i j k l}}$ for the ideal isotropic material as:

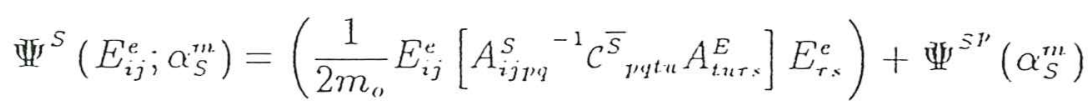


Substituting eq.(15) into eq.(12) leads to the secant constitutive equation in the real anisotropic space defined in terms of the stress field in the ideal isotropic fictitious space as:

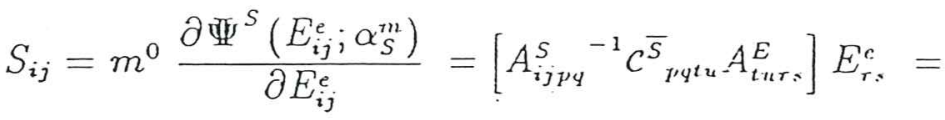

$$
\begin{aligned}
& =A_{i j p q}^{S}{ }^{-1} C^{\bar{S}}{ }_{p q t u} \bar{E}_{t t u}^{e}=A_{i j \gamma^{\prime}}^{S}{ }^{-1} \bar{S}_{p q}
\end{aligned}
$$

where $\bar{S}_{i j}=\mathcal{C}_{i j k l}^{\bar{S}} \bar{E}_{k j}^{c}$ are the stresses in the fictitious isotropic space. Eq. (16) confirms the assumptions made in eqs. (5) and (7).

II.3 .- Flow rule. Evolution of the int ?rnal variables.

From the transformation rules expressed by eqs.(5) and (7) and the definition of the plastic potential function in the fictitious isotropic space (eq.(10)), the flow rule and the evolution of the internal plastic variables $\alpha_{S}^{m}$ are obtained in the form

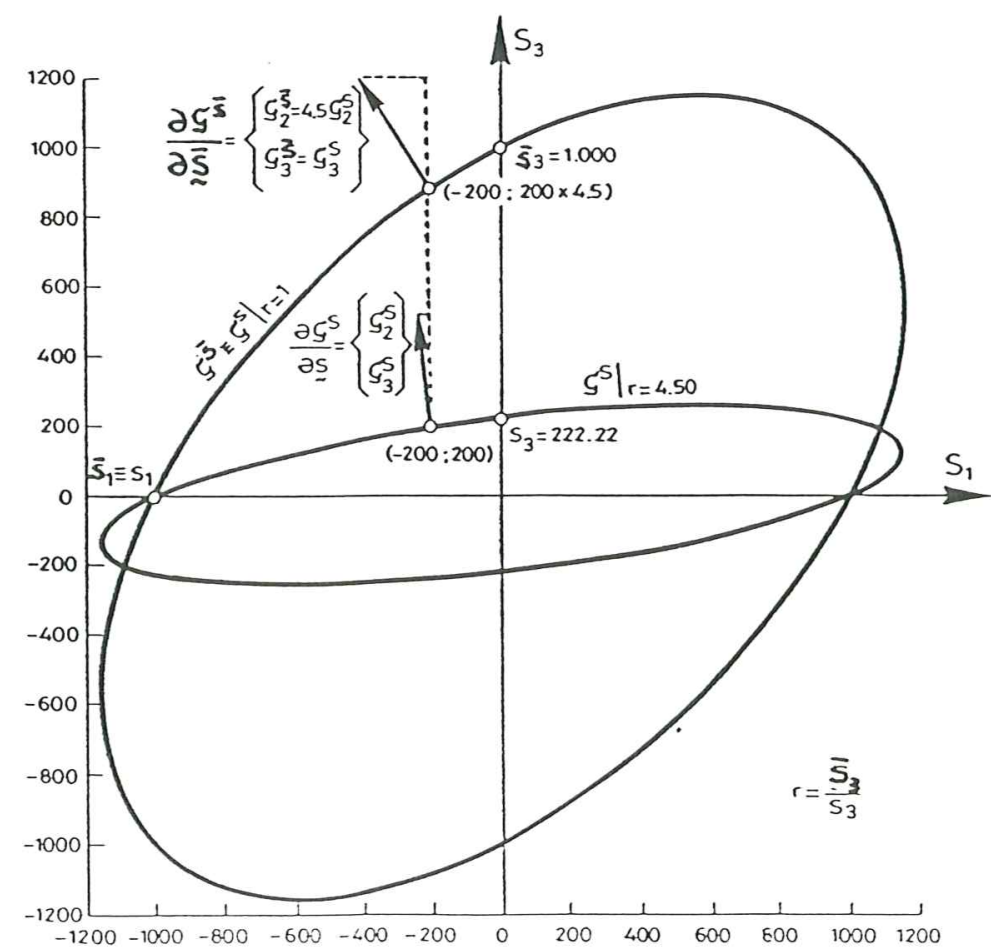

Figure 3: Differences in the plastic flow o: the real $(r=1)$ and fictitious spaces $(r=4.5)$ 


$$
\begin{aligned}
\dot{E}_{i j}^{p} & =\dot{\lambda} \frac{\partial \mathcal{G}^{S}}{\partial S_{i j}}=\dot{\lambda} \frac{\partial \mathcal{G}^{\bar{S}}}{\partial \bar{S}_{k l}} \frac{\partial \bar{S}_{k l}}{\partial S_{i j}}=\dot{\lambda} \underbrace{\overbrace{\frac{\partial \mathcal{G}_{k l}^{\bar{S}}}{\partial \bar{S}_{k l}}}^{\bar{S}_{l: l i j}^{S}}}_{R_{i j}^{S}}=\left(\dot{E}_{k l l}^{\prime}\right)^{\bar{S}} A_{k l i j}^{S} \\
\dot{\alpha}_{S}^{m} & =\dot{\lambda}\left(h_{i j}^{m}\right)_{S} \frac{\partial \mathcal{G}^{S}}{\partial S_{i j}}=\dot{\lambda}\left(h_{i j}^{m}\right)_{S} \frac{\partial \mathcal{G}^{\bar{S}}}{\partial \bar{S}_{k l}} \frac{\partial \bar{S}_{k l}}{\partial S_{i j}}=\dot{\lambda}\left(h_{i j}^{m}\right)_{S} \underbrace{\frac{\partial \mathcal{G}^{\bar{S}}}{\partial \bar{S}_{k l}} A_{k l i j}}_{R_{i j}^{S}} \\
& =\dot{\lambda}\left(h_{k l}^{m}\right)_{\bar{S}} \underbrace{R_{k l}^{\bar{S}}}_{\frac{\partial \mathcal{G}^{\bar{S}}}{\partial \bar{S}_{k l}}}=\dot{\alpha}_{\frac{m}{S}}^{m}
\end{aligned}
$$

Due to the additivity strain concept eq. (14), the elastic strains tranformation rule is extended to the plastic strains, i.e..

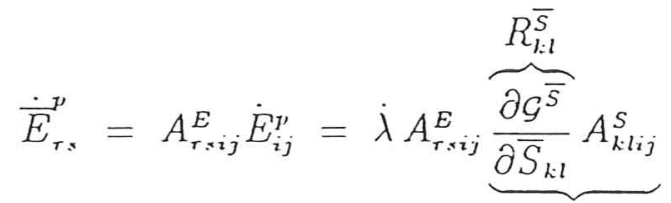

$$
\begin{aligned}
& \underbrace{R_{i j}^{S}}_{R_{r s .}^{S}}
\end{aligned}
$$

where $\left(h_{i j}^{m}\right)_{S}$ and $\left(h_{i j}^{m}\right)_{\bar{S}}$ are tensorial functions to be determined 13,16 for each of the " $m$ " internal variables involved, $R_{k l}^{\bar{S}}$ is the plastic flow in the fictitious stress space, as shown in Figure $3, R_{i j}^{S}$ is the plastic flow in the real stress space, $R_{r s .}^{S}$ is the plastic flow in the fictitious stress space, $R_{k l}^{\bar{s}}$ is the plastic flow in the fictitious isotropic space and $\dot{\bar{E}}_{r \text { s }}^{\prime \prime}$ is the fictitious plastic strain. 
II.4 -- Free energy in the fictitious isotropic space. Uniqueness of the dissipation.

Note that the dissipated mechanical power $\Xi_{m c c \text {. }}^{s}$ can be written in the fictitious isotropic space $\Xi_{\text {mec }}^{\bar{S}}$. by substituting the flow rule, the evolution law for the internal variables (eqs.(17)) and the transformation spaces rules (eqs. (5) and (7)) into eq. (10), i.e.*:

$$
\begin{aligned}
\Xi_{m e c .}^{S}= & \underbrace{\left(\frac{S_{i j}}{m^{0}}-\frac{\partial \Psi^{S}}{\partial E_{i j}^{e}}\right) \dot{E}_{i j}^{e}}_{=0 \text { (Colleman) }}+\frac{S_{i j} \dot{E}_{i j}^{p}}{m^{0}}-\sum_{m} \frac{\partial \Psi^{S}}{\partial \alpha_{S}^{m}} \dot{\alpha}_{S}^{m}= \\
= & \frac{\bar{S}_{i j}\left(\dot{E}_{i j}^{p}\right)^{\bar{S}}}{m^{0}}-\sum_{m} \frac{\partial \Psi}{\partial \alpha_{\frac{1 m}{S}}} \dot{\alpha}_{\frac{m}{S}}^{\bar{s}} \equiv \Xi_{\text {mec. }}^{\bar{S}} \geq 0
\end{aligned}
$$

It is deduced from eq.(19) that the dissipation is an invariant of the thermodynamic process and, therefore, its value is independent

* REMARK: The plastic potential transformation is derived in the following manner:

$$
\begin{aligned}
& S_{i j} \dot{E}_{i j}^{p}=\left(A_{i j \tau s}^{S}{ }^{-1} \bar{S}_{\tau s}\right)\left(A_{i j k l}^{E}{ }^{-1} \dot{\bar{E}}_{k l}^{\prime \prime}\right)= \\
& =\dot{\lambda} A_{i j r s}^{S}{ }^{-1} \bar{S}_{\tau s} A_{i j k l}^{E}{ }^{-1} A_{k l t u t u}^{E} \frac{\partial \mathcal{G}^{\bar{S}}}{\partial \bar{S}_{n p}} A_{n p t u l}^{S}= \\
& =\dot{\lambda} A_{i j r s}^{S}{ }^{-1} \bar{S}_{r s} I_{i j t u} \frac{\partial \mathcal{G}^{\bar{S}}}{\partial \bar{S}_{n p}} A_{n p_{1} t u}^{S}= \\
& =\dot{\lambda} A_{i j r s}^{S}{ }^{-1} \bar{S}_{r s} \frac{\partial \mathcal{G}^{\bar{S}}}{\partial \bar{S}_{n p},} A_{n p i j}^{S}=\dot{\lambda} \bar{S}_{r s s} \frac{\partial \mathcal{G}^{\bar{S}}}{\partial \bar{S}_{n p p}} I_{n p j r s s}= \\
& =\dot{\lambda} \bar{S}_{n_{p} p} \frac{\partial \mathcal{G}^{\bar{S}}}{\partial \bar{S}_{n p}}=\bar{S}_{n p}\left(\dot{E}_{i j}^{p}\right)^{\bar{S}} .
\end{aligned}
$$


of the space where it is computed. Also, the free energy can be obtained in the fictitious isotropic space in the form:

$$
\Psi^{\bar{S}}\left(\bar{E}_{i j}^{e} ; \alpha \frac{m}{S}\right)=\left(\frac{1}{2 m_{o}} \bar{E}_{i j}^{e} c^{\bar{S}_{i j k l}}{\overline{E_{k l}^{c}}}^{c}\right)+\Psi^{\bar{S}^{p}}\left(\alpha \frac{m}{\bar{S}}\right)
$$

Eq. (20) is equivalent to expression (13) defined in the real anisotropic space, and therefore the constitutive equation given by eq.(16) still holds. This is.

$$
\begin{aligned}
& \bar{S}_{i j}=m^{0} \frac{\partial \Psi \bar{s}\left(\bar{E}_{i j}^{e} ; \alpha_{S}^{m}\right)}{\partial \bar{E}_{i j}^{c}}=c^{\bar{S}_{i j k l}} \bar{E}_{k l}^{c}= \\
& =\left(A_{i j r s}^{S} C^{S}{ }_{r: s t u} A_{t u l k l}^{E}{ }^{-1}\right)\left(A_{k l l_{p q}}^{E} E_{p^{\prime} q}^{e}\right)= \\
& =A_{i j r s}^{S} \mathcal{C}^{S}{ }_{r s t u} I_{t u p q} E_{p q}^{e}= \\
& =A_{i j r s}^{S} \mathcal{C}^{S}{ }_{r s t u} E_{t u}^{e}=A_{i j r s}^{S} S_{T s}
\end{aligned}
$$

where the stress transformation rule is recovered.

\section{II.5 .- Tangent constitutive equation.}

The rate form of the constitutive equation is obtained by performing the temporal derivative of the secant expression (16) 
as:

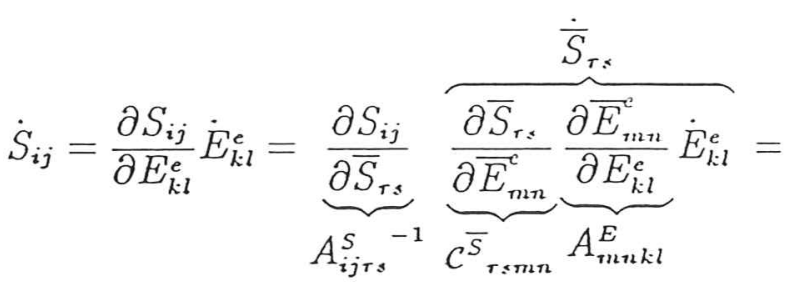

$$
\begin{aligned}
& =A_{i j r s}^{-1} c_{r s m n n}^{\bar{S}} A_{m m k l}^{E} \dot{E}_{k l}^{e}=A_{i j r s}^{-1} \underbrace{\mathcal{C}_{r s m n l}^{\bar{S}} A_{m n k l}^{E}\left(\dot{E}_{k l}-\dot{E}_{k l}^{r^{\prime}}\right)}_{\dot{\bar{S}}_{r s}}= \\
& =A_{i j r s}^{-1} \underbrace{c_{r s m n n}^{\bar{S}}\left(\dot{\bar{E}}_{m 2 n}-\dot{\bar{E}}_{m n n}^{\prime \prime}\right)}_{\overline{\bar{S}}_{r s}}
\end{aligned}
$$

Eq. (22) can be interpreted as a linear transformation of the following rate constitutive equation defined in the fictitious isotropic space:

$$
\dot{\bar{S}}_{i j}=\frac{\partial \bar{S}_{i j} \dot{\bar{E}}_{k l}^{e}}{\partial \bar{E}_{k l}^{c}}=c_{i j l l}^{\bar{S}} \dot{\bar{E}}_{k l}^{e}=c_{i j k l}^{\bar{S}}\left(\dot{\bar{E}}_{k l}-\dot{\bar{E}}_{k l}^{\prime}\right)
$$

The plastic consistency condition leads to the standard rate form of the constitutive equation in the fictitious isotropic space as:

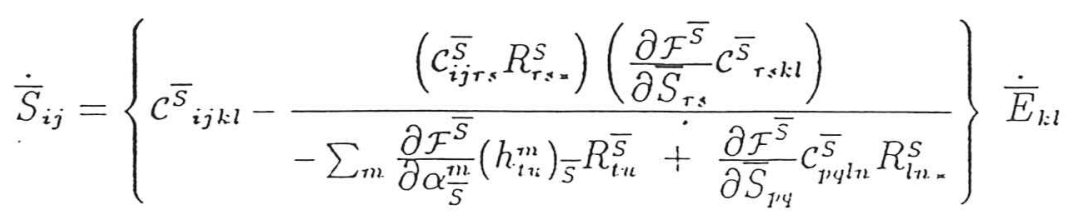


or in compact form:

$$
\dot{\bar{S}}_{i j}=\mathcal{c}^{\bar{S}_{i j k l}^{p p}} \dot{\bar{E}}_{k l}
$$

Combining now (22) and (23) leads to the final expression of the rate constitutive equation in the real anisotropic solid as:

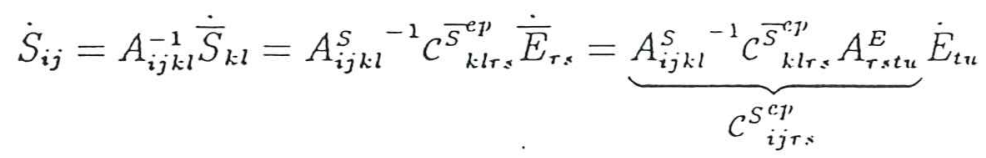

Therefore, the constitutive model requires only the following definitions in each of the two spaces used:

- real anisotropic space $:\left\{\begin{array}{l}\text { initial constitutive tensor }\left(\mathcal{C}^{S}{ }_{i j k l}\right)_{l o c u l} \\ \text { yield strength } \quad\left(f_{i j}^{S}\right)_{l o c u l}\end{array}\right.$

$$
\text { - fictitious isotropic space }: \begin{cases}\text { initial fictictitious constitutive tensor } & C_{i j k l}^{\bar{S}} \\ \text { yield function } & \mathcal{F}^{\bar{S}}\left(\bar{S}_{i j}, \alpha_{\bar{S}}^{m}\right)=0 \\ \text { potential function } & \mathcal{G}^{\bar{S}}\left(\bar{S}_{i j}, \alpha \frac{m}{\bar{S}}\right)=\mathcal{K} \\ \text { yield strength } & \left(f_{i j}^{\bar{S}}\right)\end{cases}
$$

Above definitions allow to derive all the basic constitutive relationships, including the evolution of the inner variables, necessary to formulate in a precise manner the constitutive behaviour of an anisotropic elasto-plastic solid by means of an associated fictitious isotropic solid. 


\section{III.- INTEGRATION OF THE CONSTITUTIVE EQUATION.}

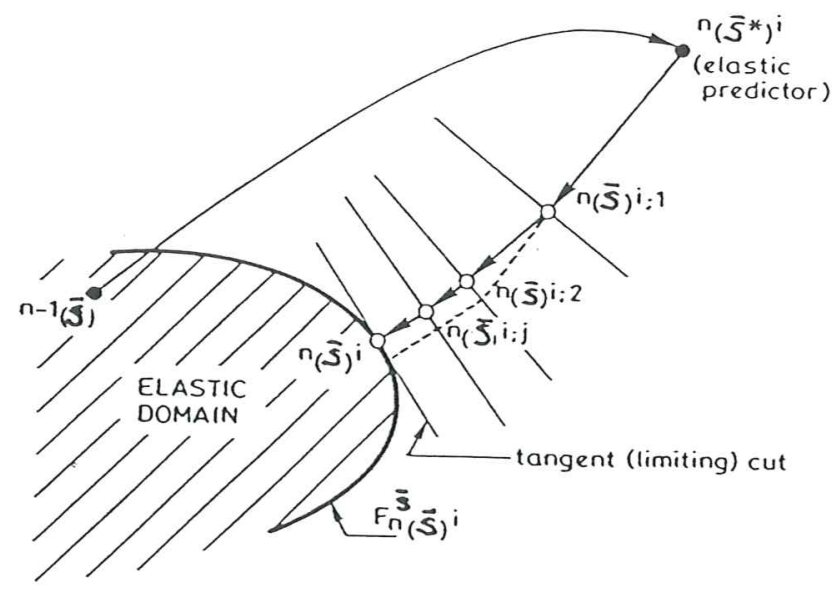

Figure 4: Plastic flow definition in the cutting plane algorithm ${ }^{18}$.

The constitutive equation is integrated by means of a tangent cutting plane algorithm as presented by Ortiz and Simo ${ }^{18}$. This kind of Euler-backward algorithm is used for integration of rate constitutive equations in orthotropic solids ". Nevertheless, in our case, note that the integration of the constitutive equation is done on the isotropic fictitious space. The integration methodology is based in the "mapping return" concept, i.e.: the return path towards the converged yield function follows the direction of the normal flow computed at each iterative solution (see Fig. 4). Further details of this technique can be found in ${ }^{4,5,19}$

\section{IV.- NUMERICAL IMPLEMENTATION OF THE ANISOTROPIC ELASTO-PLASTIC MODEL PROPOSED.}

The basic steps for implementing the anisotropic model proposed into standard elasto-plastic finite element programs ${ }^{24}$ are given next. 
- LOOP OVER SOLUTION INCREMENT: $n=1, N I N C R$ ITERATION LOOP: $i=1, N I T E R$ IF (n.GT.1 .OR. i.GT.1) GOTO 4 1.- Define stiffness and rotation tensors:

$$
f^{\bar{S}} ;\left(f^{S}\right)_{\text {locul }} ; \mathcal{C}^{\bar{S}} ;\left(\mathcal{C}^{S}\right)_{\text {locul }} ; \mathcal{R}
$$

2.- Obtain the spaces transformation tensors:

$$
\begin{aligned}
& f^{S}=\mathcal{R}:\left(f^{S}\right)_{\text {local }} \\
& A^{S}=f^{\bar{S}} \otimes f^{S} \\
& \mathcal{C}^{S}=\mathcal{R}:\left(\mathcal{C}^{S}\right)_{\text {local }}: \mathcal{R} \\
& A^{E}=\overline{\mathcal{C}}^{-1}: A^{S}: \mathcal{C}^{S}
\end{aligned}
$$

3.- Initialice the isotropic constitutive tensor:

$$
{ }^{1}\left(\mathcal{c}^{\bar{S}^{c p}}\right)^{0}={ }^{1}\left(\mathcal{c}^{\bar{S}}\right)^{0}
$$

4.- Compute tangent stiffness:

$$
\begin{gathered}
n\left(\mathcal{C}^{S^{e p}}\right)^{i-1}=A^{S^{-1}}:{ }^{n}\left(\mathcal{C}^{\bar{S}^{e p}}\right)^{i-1}: A^{E} \\
n\left(K^{(e)}\right)^{i-1}=\int_{V} B:^{n}\left(\mathcal{C}^{S^{c p}}\right)^{i-1}: B d V \\
n(K)^{i-1}=\mathrm{A}_{e=1}^{n e}{ }^{n}\left(K^{(e)}\right)^{i-1}
\end{gathered}
$$


5.- Compute displacements and strains:

$$
\begin{aligned}
& n(\delta U)^{i}={ }^{n}\left(K^{-1}\right)^{i-1} \cdot{ }^{n}\left(F_{\text {Texid }}\right)^{i-1} \\
& n(\Delta U)^{i}={ }^{n}(\Delta U)^{i-1}+{ }^{n}(\delta U)^{i} \\
& n(\Delta E)^{i}=f^{n}(\Delta U)^{i} \\
& n(E)^{i}={ }^{n-1}(E)+{ }^{n}(\Delta E)^{i} .
\end{aligned}
$$

6.- Evaluate predicted stresses:

$$
\begin{aligned}
& n\left(\Delta S^{*}\right)^{i}={ }^{1}\left(\mathcal{C}^{S}\right)^{0}:^{n}(\Delta E)^{i} \\
& { }^{n}\left(S^{*}\right)^{i}={ }^{n-1}(S)+{ }^{n}\left(\Delta S^{*}\right)^{i}
\end{aligned}
$$

7.- Transform predicted stresses to the fictitious isotropic space:

$$
{ }^{n}(\bar{S})^{i}=A^{S}:{ }^{n}\left(S^{*}\right)^{i}
$$

8.- Integrate isotropic constitutive law: ITERATION LOOP: $\mathrm{j}=1$,JITER

$$
\text { for: } j=1 \Longrightarrow "(\bar{S})^{i, 0}={ }^{n}\left(\bar{S}^{\prime}\right)^{i} \quad ; \quad "\left(\Delta \bar{E}^{\prime \prime}\right)^{i .0}=0
$$




$$
\begin{aligned}
\text { - } 8.1 & { }^{n}(\bar{S})^{i, j}={ }^{n}(\bar{S})^{i, j-1}-{ }^{1}\left(\mathcal{C}^{S}\right)^{0}:^{n}\left(\Delta \bar{E}^{\prime}\right)^{i, j-1} \\
& \text { If : } \mathcal{F}^{\bar{S}}\left(\bar{S} ; \alpha \frac{m}{S}\right)_{n \bar{S}^{i, j-1}} \leq 0\left\{\begin{array}{l}
\text { and } j=1 \Longrightarrow \text { GOTO } 10 \\
\text { and } j>1 \Longrightarrow \text { GOTO } 9
\end{array}\right.
\end{aligned}
$$

else :

$$
\begin{aligned}
& { }^{n}\left(\Delta \bar{E}^{p}\right)^{i, j}=(\Delta \lambda)^{j} \cdot A^{E}:{ }^{n}\left(\frac{\partial \mathcal{G}^{\bar{S}}}{\partial \bar{S}}\right)_{n, \bar{S}^{i, j}}^{j}: A^{S}=(\Delta \lambda)^{j} \cdot{ }^{n}(R)_{n \bar{S}_{0}^{i, j}}^{j} \\
& n\left(\bar{E}^{p}\right)^{i, j}={ }^{n}\left(\bar{E}^{p}\right)^{i, j-1}+{ }^{n}\left(\Delta \overline{E^{p}}\right)^{i, j} \\
& { }^{n}\left(\Delta \alpha_{\frac{m}{S}}\right)^{i, j}=(\Delta \lambda)^{j} \cdot{ }^{n}\left(h^{m}\right)^{i, j}:{ }^{n}\left(\frac{\partial \mathcal{G}^{\bar{S}}}{\partial \bar{S}}\right)_{r \cdot \bar{S}^{i, j}}^{j} \\
& { }^{n}\left(\alpha^{\frac{m}{S}}\right)^{i, j}={ }^{n}\left(\alpha^{m}\right)^{i, j-1}+{ }^{n}\left(\Delta \alpha^{m}\right)^{i, j} \\
& j=j+1 \text { Go back to } 8.1 \text {. }
\end{aligned}
$$

9.- Compute tangent constitutive tensor in the isotropic space:

$$
{ }^{n}\left(\mathcal{C}^{\bar{S}^{e p}}\right)^{i}=\left\{\mathcal{C}^{\bar{S}}-\frac{\left(\mathcal{C}^{\bar{S}}: R_{-}^{S}\right) \otimes\left(\frac{\partial \mathcal{F}^{\bar{S}}}{\partial \bar{S}} \mathcal{C}^{\bar{S}}\right)}{-\sum_{m} \frac{\partial \mathcal{F}^{\bar{S}}}{\partial \alpha_{\bar{S}}}\left(h^{\frac{m}{S}}\right): R^{\bar{S}}+\frac{\partial \mathcal{F}^{\bar{S}}}{\partial \bar{S}} \mathcal{C}^{\bar{S}}: R_{*}^{S}}\right\}_{n \bar{S}^{i}}
$$

10.- Back transformation of stresses to the real anisotropic space:

$$
n(S)^{i}=A^{S^{-1}}:{ }^{n}(\bar{S})^{i}
$$

11.- Compute residual forces:

$$
{ }^{n}\left(F_{\text {residl }}^{(e)}\right)^{i}=\int_{V} B:^{n}(S)^{i} d V-f_{\text {ext }}
$$




$$
\begin{aligned}
& n\left(F_{\text {resid }}\right)^{i}=\mathrm{A}_{e=1}^{n e} n\left(F_{\text {residd }}^{(e)}\right)^{i} \\
& \text { Is }\left\|F_{\text {resid }}\right\|>0 ? \Longrightarrow i=i+1 \text {. Go back to } 4 \\
& \quad \text { else : }
\end{aligned}
$$

Converged solution for the $n t h$ increment.

$$
n=n+1
$$

Compute new incremental solution

V.- EXAMPLES.

- V-1. Orientation effect of fibers in a composite.

Let us cunsider the case where continuous glass fibers are placed onedirectionally within an epoxy matrix material and form an angle $\phi$ with the applied tension load as shown in Figure 5 . The tension failure limit strength $\left(S_{y}\right)$ of the composite depends on the fiber orientation which angle will be varied between $0^{\circ}$ and $90^{\circ}$. In order to establish strength and failure characteristics of the material, it is necessary to consider a number of fundamental properties related to the composite behaviour such as: Young modulus along principal directions: in-plane directions, $E_{\text {Long. }}=591998.8 \mathrm{~kg} / \mathrm{cm}^{2}$, and $E_{\text {Trans. }}=140617.3 \mathrm{~kg} / \mathrm{cm}^{2}$. Poisson ratio: $\nu_{L T}=0.293$. Elastic strength limits, in-plane directions: for the longitudinal fiber behaviour $f_{\text {Long }}^{\text {Comp. }}=19686.4 \mathrm{~kg} / \mathrm{cm}^{2}, f_{L m g}^{T \text { ens. }}=9561.9 \mathrm{~kg} / \mathrm{cm}^{2}$, $f_{L T}^{\text {Shear. }}=421.8 \mathrm{~kg} / \mathrm{cm}^{2} ;$ and for the transverse fiber behaviour $f_{\text {Trans }}^{\text {Comp. }}=1406.2 \mathrm{~kg} / \mathrm{cm}^{2}, f_{\text {Trans }}^{\text {Tens. }}=281.2 \mathrm{~kg} / \mathrm{cm}^{2}$.

Additional data required defining the fictitious isotropic model are: equivalent Young modulus: $E^{\bar{s}}=591998.8 \mathrm{~kg} / \mathrm{cm}^{2}$, equivalent elastic compression strength limit: $f^{t a u}=19686.4 \mathrm{~kg} / \mathrm{cm}^{2}$, MohrCoulomb yield function, fiber plastic flow (it only has components on the fiber direction) and perfect plasticity. Figure 5 shows the tension strength limit $\left(S_{y} / f_{T}\right.$ where $f_{T}$ is the tension failure limit strength of the fibers) of the composite as the fiber orientations 
angle $\phi$ varies. Note that the maximum strength takes place for $\phi=0^{\circ}$ and the minimum for $\phi=45^{\circ}$. The material strength is greater for $\phi=90^{\circ}$ than for $\phi=45^{\circ}$ due to the transversal deformation restricted by the compression fiber strength of the fibers.

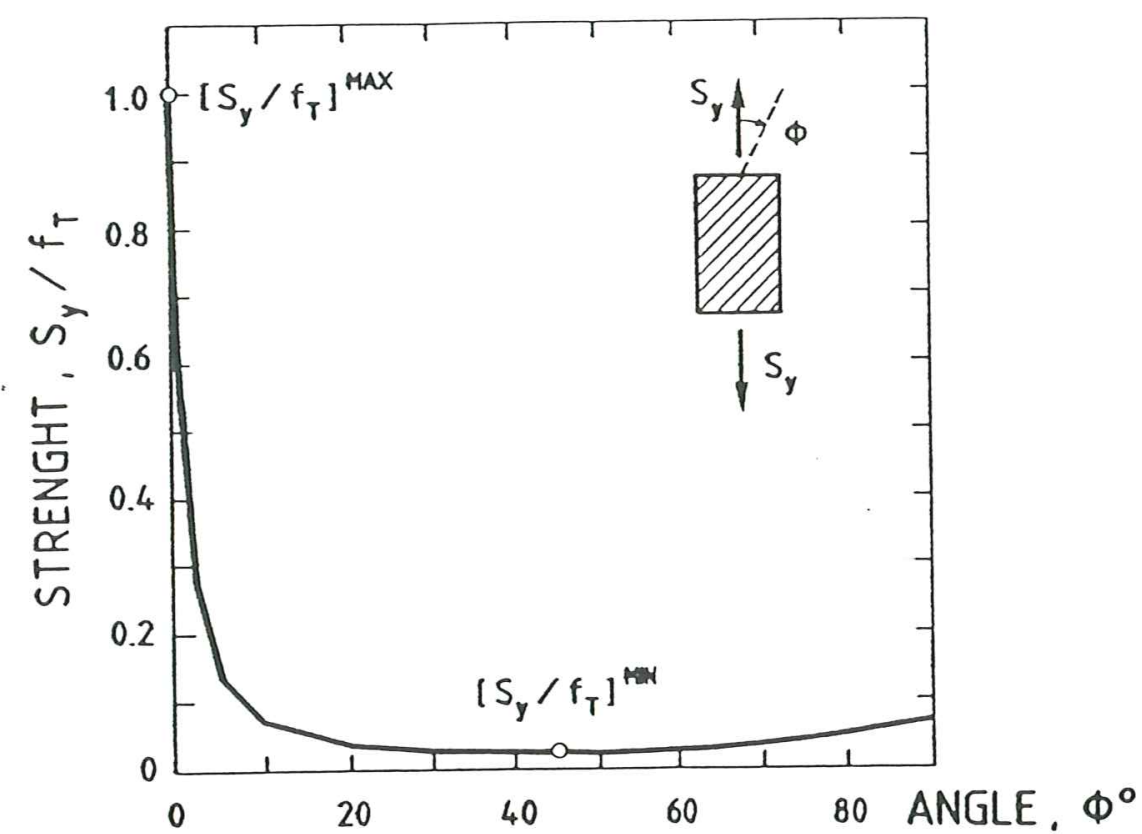

Figure 5: Strength ratio Vs. fiber slope angle, for an epoxy matrix with glass fiber.

The particular form of the curve in Figure 5, depends on the yield and potential plastic functions adopted in the fictitious isotropic solid. Here the Mohr-Coulomb yield function was arbitrary chosen.

- V-2. A comparison with Mises-Hill orthotropic model.

The anisotropic model proposed has been applied to the analysis of a rectangular specimen under axial loading acting along three different directions (Fig. 6). Plane stress conditions have been assumed. The geometry has been discretized using a simple mesh of sixteen standard 4 nodes quadrilateral elements as shown in Figure 6. Numerical results obtained have been compared with those provided by the well known orthotropic Mises-Hill model ? 


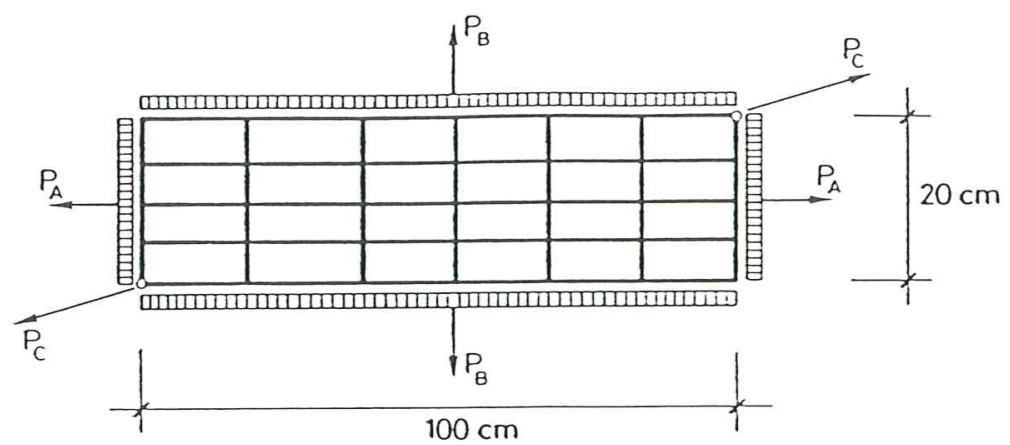

Figure 6: Test specimen. Geometry, boundary condition, loading and finite element mesh.

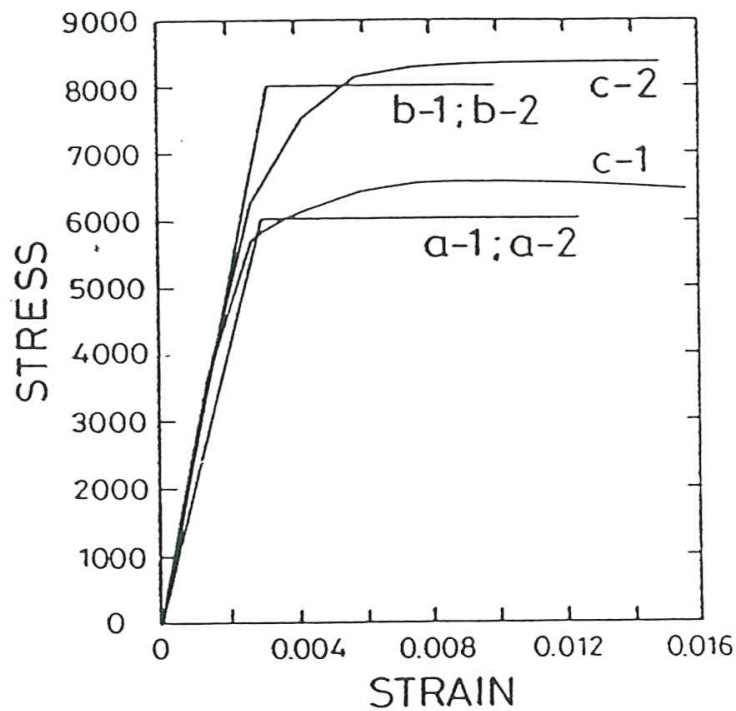

Figure 7: Strain-stress curves at center of the sample for perfect plasticity using the anisotropic model proposed and the Mises-Hill model. Loading(a) : a-1 Present model, a-2 Mises-Hill model; Loading (b) : b-1 Present model, b-2 Mises-Hill model; Loading (c) : c-1 Present model, c-2 Mises-Hill model.

The material properties are the following:

Young modulus along principal directions: In-plane directions, $E_{1}=2.5 E^{6} \mathrm{~kg} / \mathrm{cm}^{2}$, and $E_{2}=2.0 E^{6} \mathrm{~kg} / \mathrm{cm}^{2}$. Transverse direction $E_{3}=2.0 E^{6} \mathrm{~kg} / \mathrm{cm}^{2}$. Shear modulus: $G=0.45 E^{6} \mathrm{~kg} / \mathrm{cm}^{2}$. Poisson ratio: $\nu=0.3$. Elastic strength limits: In-plane directions: $f_{1}=8000.0 \mathrm{~kg} / \mathrm{cm}^{2}$ and $f_{2}=6000.0 \mathrm{~kg} / \mathrm{cm}^{2}$, transverse direction $f_{3}=6000.0 \mathrm{~kg} / \mathrm{cm}^{2}$.

Additional data defining the fictitious isotropic model are: Equivalent Young modulus: $E^{\bar{s}}=2.5 E^{6} \mathrm{~kg} / \mathrm{cm}^{2}$, Equivalent 


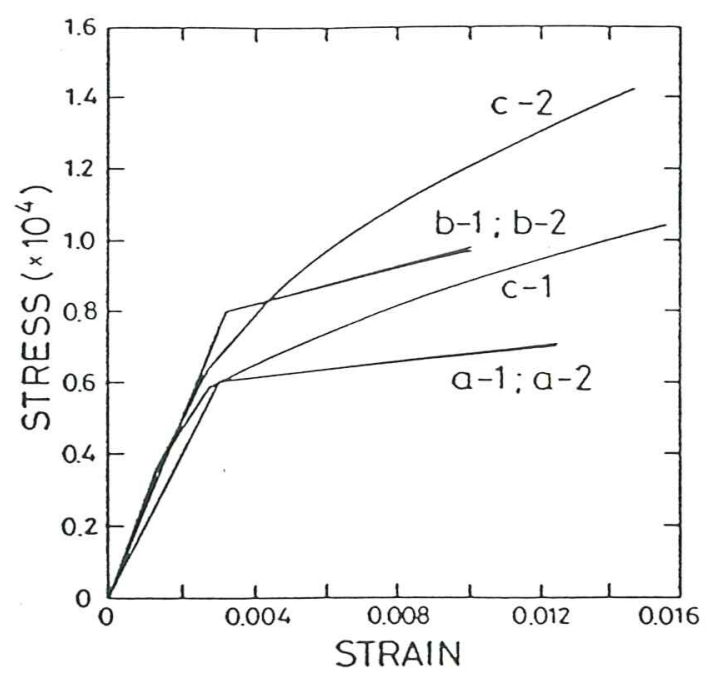

Figure 8: Strain-stress curves at center of the sample for hardening plasticity case using the anisotropic model proposed and the Mises-Hill model. Loading (a) : a-1 Present model, a-2 Mises-Hill model; Loading (b) : b-1 Present model, b-2 Mises-Hill model; Loading (c) : c-1 Present model, c-2 Mises-Hill model.

elastic strength limit: $f^{t a u}=8000.0 \mathrm{~kg} / \mathrm{cm}^{2}$, and Von-Mises yield function.

Figure 6 shows the three loading types applied: (a) axial loading parallel to the maximum strength direction: (b) transverse loading orthogonal to the maximum strength direction, and (c) diagonal loading.

Figures 7 and 8 show the comparison between the strain-stress curves obtained at the center of the sample, assuming perfect plasticity and hardening plasticity with a hardening modulus $H=$ $250000.0 \mathrm{~kg} / \mathrm{cm}^{2}$, respectively.

Note that results obtained with both models coincide for loading cases (a) and (b). However, considerable differences arise for the diagonal loading case (c). This is due to the excessive influence of shear in Mises-Hill theory leading to over-stiff results. The values obtained with the proposed model are within reasonable limits and bounded by those of loading cases (a) and (b) as expected. 
- V-3. Analysis of a fiber reinforced composite.

The third example is the analysis of a plane rectangular specimen of a fiber reinforced composite material. Figure 9 shows the specimen geometry, the boundary conditions and the finite element mesh of sixteen 4 node quadrilateral elements used. A prescribed longitudinal displacement has been imposed to both ends of the specimen as shown in Figure 9.

The specimen is composed of an isotropic matrix and a $20 \%$ of long fibers. Initially the fibers are considered aligned along the longitudinal direction and then along the transverse direction. The combined effect of fiber and matrix material has been modelled using a multiphase model based an mixing theory developed by the authors ${ }^{25}$

Material properties: Matrix isotropic material: $E=7.24 \mathrm{tn} / \mathrm{mm}^{2}, \nu=0.33$, limit elastic strength $f=0.036 \mathrm{tn} / \mathrm{mm}^{2}$ and $80 \%$ of fraction volume participation. Isotropic associated Von-Mises plasticity has been assumed in this case.

Fibers. The anisotropic model proposed has been used with $E=84.4 \mathrm{tn} / \mathrm{mm}^{2}$ and limit elastic strength $f=0.2283 \mathrm{tn} / \mathrm{mm}^{2}$, $20 \%$ of fraction volume participation. Von-Mises yield function and plastic flow along the fiber direction has been assumed in the fictitious isotropic space.

Perfect plastic behaviour for both materials has been assumed.

Figure 10-1 displays the stress-strain behaviour for the composite with longitudinal fibers, showing the behaviour of the composite (curve b), the matrix (curve c) and the fibers (curve a). Also, the same figure shows that the stiffness of the composite remain equal to that of the matrix after plastification of the fibers.

Figure 10-2 shows the stress-strain behaviour for the composite 
a)

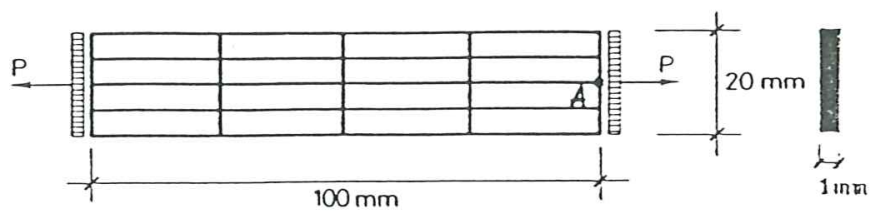

b)

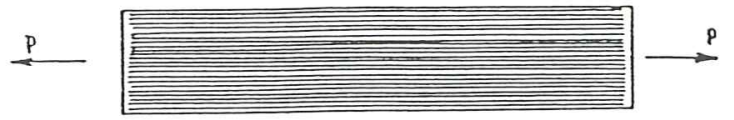

c)

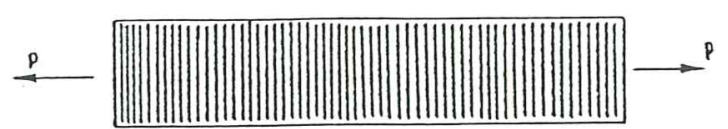

Figure 9: Fiber reinforced specimen. Geometry, boundary condition, loading and finite element mesh.

with transversal fibers. Curve (a) shows the evolution of the longitudinal stress in the composite, curve (b) shows that the strength of the matrix is greater than that of the composite. This can be explained by the small contribution of the transverse fibers to the global longitudinal stiffness. Therefore, the resisting material has $20 \%$ less transverse cross area with the corresponding reduction in stiffness. Curve (c) in the same figure shows the transversal tension stress in the matrix and the corresponding compression stress in the fibers (curve d). Finally curve (e) shows the null "global" resistence of the composite in the transverse direction as espected.

Figure 11 shows the load-displacement curves for the two fiber orientations studied.

Figures 12 and 13 show, in qualitative form, the principal stress field in the composite material, as well as in the matrix and the fiber components, for each of two fiber orientation cases analyzed, respectively. 


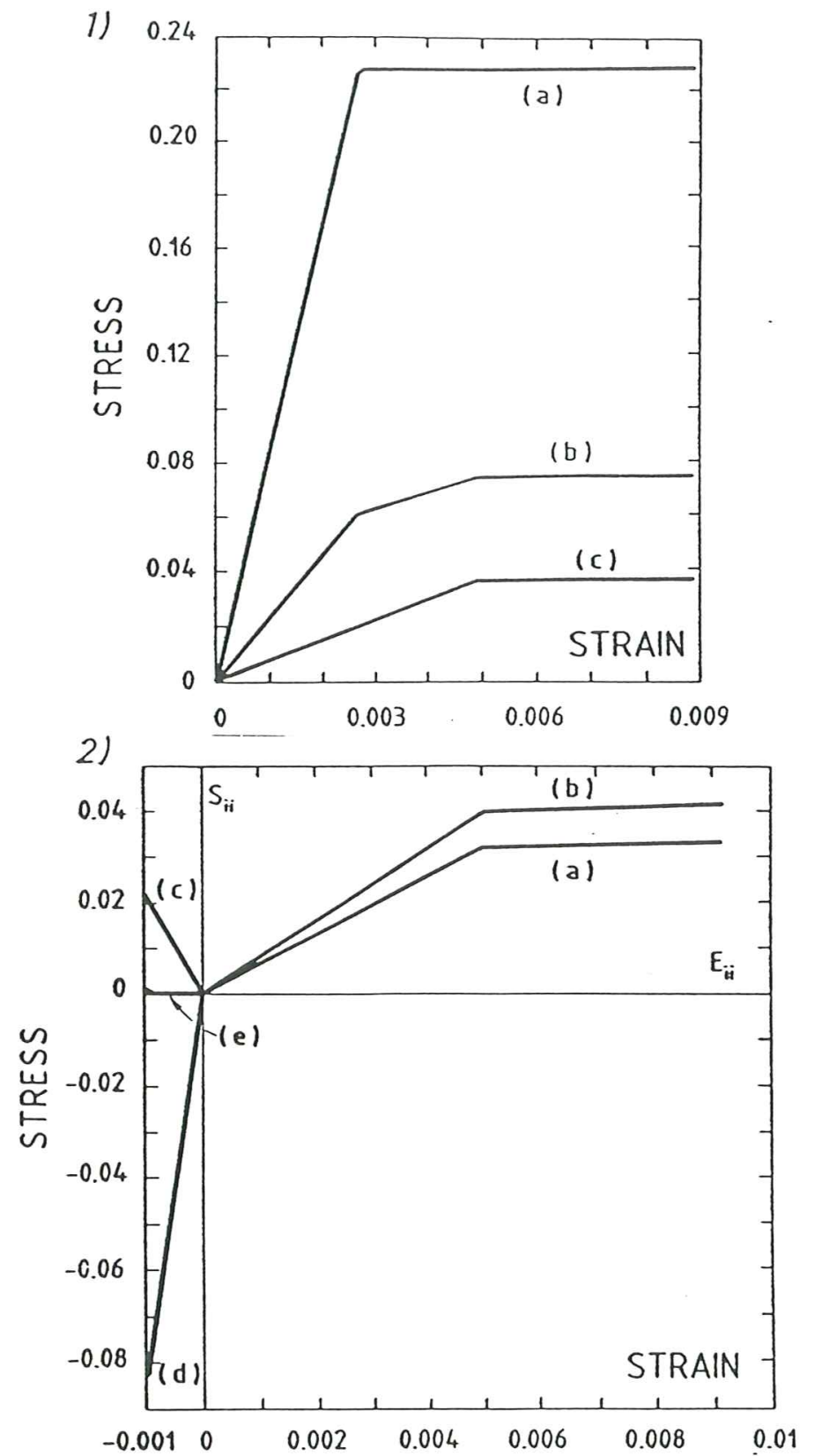

Figure 10 Fiber reinforced composites: Stress-strain curves in the center of the specimen. Case (1) : Longitudinal fibers. Behaviour on the longitudinal direction of the: fibers (curve a), composite (curve b) and matrix (curve c). Case (2) : Transverse fibers. Behaviour on the longitudinal direction of the: composite (curve a) and matrix (curve b). Behaviour on the transverse direction of the: matrix (curve c), fibers (curve d) and composite (curve e).

The numerical results obtained are in good agreement with the expected values, thus showing the ability of the model proposed to analyze fiber reinforced composites. 


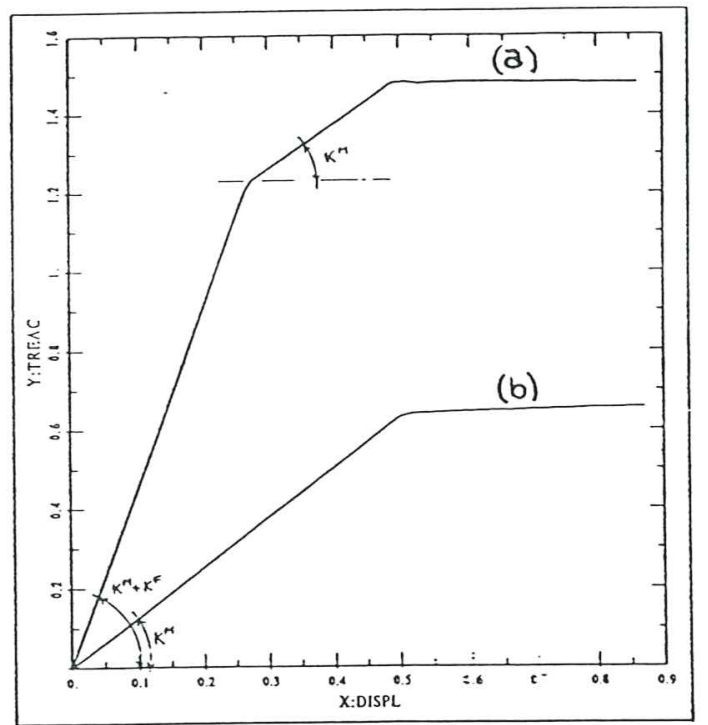

Figure 11: Load-displacement curves in point "A" of the specimen. Curve (a): Longitudinal fibers. Curve (b): Transverse fibers.

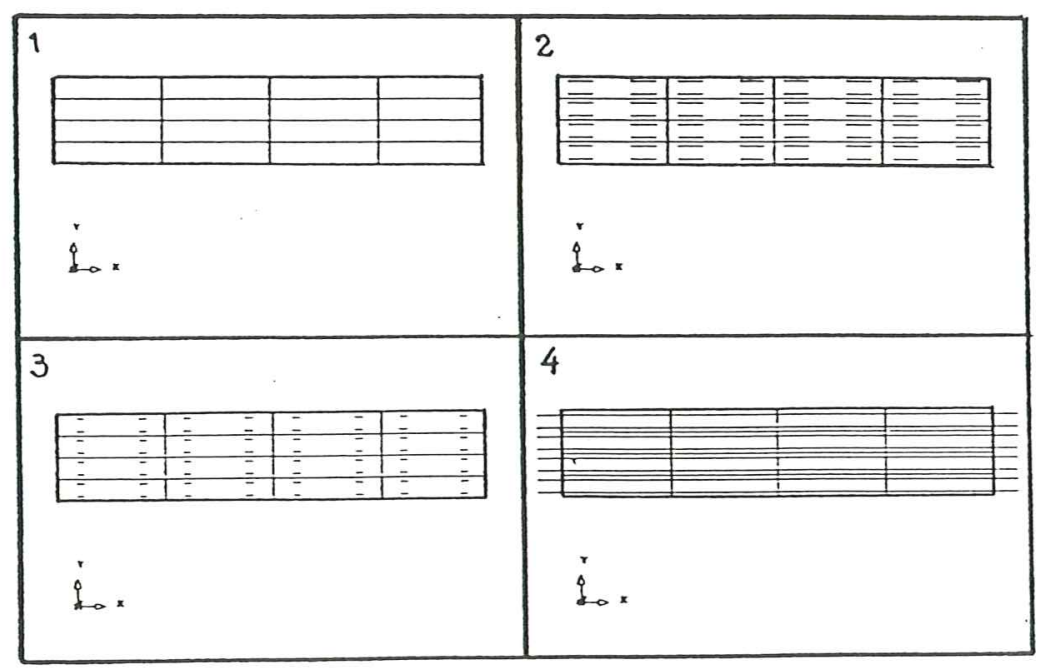

Figure 12: Principal stresses for the longitudinal fiber case. 1- Finite element mesh; 2Principal stresses in the composite; 3 - Principal stresses in the matrix; 4- Principal stresses in the fibers.

\section{VI.- CONCLUDING REMARKS.}

It has been shown that the elasto-plastic anisotropic behaviour 


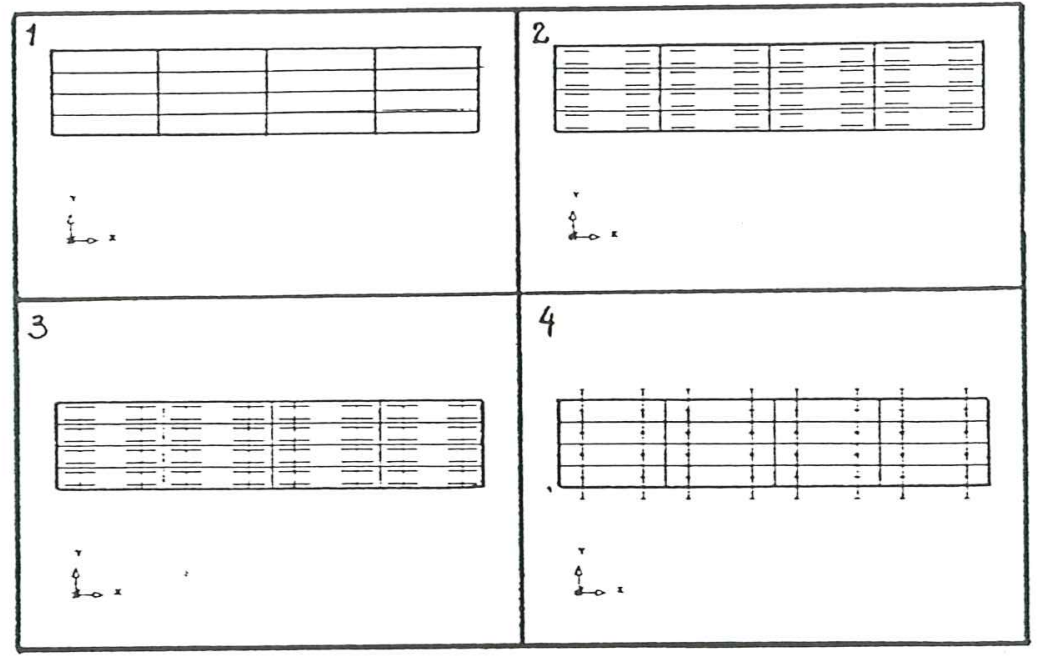

Figure 13: Principal stresses for the transverse fiber case. 1- Finite element mesh; 2Principal stresses in the composite; 3- Principal stresses in the matrix; 4- Principal stresses in the fibers.

of solids can be effectively modeled by means of an equivalent isotropic model based on standard Green-Naghdi large strain elastoplasticity theory. The model proposed is particularly suited for finite element computations of composite structures with different material properties. Initial applications of the model to standard isotropic materials and more complex fiber reinforced composites using a multiphase material model ${ }^{25}$ show promising results which will be more extensively validated in the near future. Extensions of the anisotropic formulation presented to non linear damage mechanics are straightforward and are currently investigated by the authors.

\section{VII.- REFERENCES.}

[ 1]-Betten J. -Creep Theory of Anisotropic Solids - Journal of Rheology, 25(6), pp $565-581-1981$

[2]-Better. J. -Damage Tensor in continuum mechanics -Journal de Mècanique 
théorique et appliqué - Vol, 2 No.1, pp 13,32 - 1983.

[ 3]-Betten J. -Application of Tensor Functions to the Formulation of Yield Criteria for Anisotropic Materials - International Journal of Plasticity, Vol. 4, pp 29-46 -1988 .

[ 4]-Crisfield M. A. -Non-linear finite element analysis of solids and structures - John Wiley and Sons - England 1991.

[ 5]- Feenstra P.H. and de Borst R. -Accuracy Analysis of Return Mapping Algorithms For The Hill Criterion- Proc. of 2nd. Int. Conf. on Computational Plasticity Part 1 -pp 377-388 -Ed. by D. Owen, E. Hinton and E. Oñate. Pineridge Press, 1989.

[6] - Green A. Naghdi P. -A general theory of an Elastic-Plastic Continuum. - Arch. Rational Mech. Anal., Vol. 18 - pp. 19-281 - 1964.-

[ 7]-Hill R. -The Mathematical Theory of Plasticity-Oxford Engineering Science Series - Oxford University Press - 1971 London

[ 8]- Kachanov L. - Time of the rupture process under creep conditions. -Otd Tech. Nauk. No. 8, - pp. 26-31 - 1958 .

[9]-Kothari N.C. -Dispersion hardening by powder metallurgy-Sintered Metal-Ceramic Composites, Ed. by Upadhyaya - Elsevier Science Publishers -pp. 71,85Amsterdam -1984.

[10] - Lubliner J. - On thermodynamics foundations of non-linear solid mechanics - Int. Journal non-linear Mechanics -No.7 -pp.237,254 -1972.

[11]- Lubliner J. - Thermomechanics of deformable bodies - Edited by Department of Civil Engineering University of California, Berkeley USA -1985.

[12] - Lubliner J. -Plasticity Theory - Macmillan Publishing - U.S.A - 1990

[13] - Lubliner J., Oliver J., Oller S., Oñate E. - A plastic damage model for non linear analysis of concrete - Int. Solids and Structures Vol. 25, No. 3 - pp. 299-326 $-1989$. 
[14] - Malvern L. -Introduction to the mechanics of continuous medium - Prentice Hall USA -1969

[15]- Oliver J., Cervera M., Oller S. and Lubliner J. -Isotropic damage models and smeared crack analysis of concrete-SCI-C 1990, Second Int. Conf. on Computer Aided Design of Concrete Structure, Zell am See, Austria, pp. 945-957 - 1990.

[16] - Oller S. - Un modelo de daño continuo para materiales friccionales - Tesis Doctoral Escuela Tecnica Superior de Ingenieros de Caminos Canales y Puertos de Barcelona -1988 .

[17]- Oller S. -Nuevos Materiales Estructurales, Cerámicos en Ingeniería - Centro Internacional de Métodos Numéricos en Ingeniería - Barcelona - España - 1989.

[18]- Oñate E., Oller S., Botello S., Miquel J. - Metodos avanzados de calculo de estructuras de materiales compuestos - Internal Report C.I.M.N.E. - Nro. 11, Barcelona, Enero 1991.

[19]-Ortiz M. and Simo J. - An analysis of anew class of integration algorithms for elastoplastic constitutive relations. - Int. J. Num. Meth. Eng., 23, p.353-366 1986.

[ 20]-Perić D., Dutko M., Owen D.R. - Universal anisotropic yield criterion based on superquadratic functional Representation: computational issues with applications. - Proc. of 3nd. Int. Conf. on Computational Plasticity - Part 1 -pp 77 - 102 -Ed. by D. Owen, E. Oñate and E. Hinton. Pineridge Press, 1992.

[21]- Tsai S. and Pagano N.J. - Invariant of properties of composite materials Composite materials workshop - Ed. by S. Tsai, H. Halpin and N. Pagano Technomic Publishing - Co. Ing. Stamford Conn. 1966, pp. 233 - 253

[22] - Tsai S. - Miravete A. -Diseño y análisis de materiales compuestos ed. Revertè Barcelona - Spain - 1987.

[23]-Vinson J. and Sierakowski R. - The behaviour of structures composed of composite materials - Martinus Nijhoff Publishers - 1986.

[24]-Zienkiewicz O.C. and Taylor R.L - The Finite Element Method Basic Formulation and Linear Problem - Vol.1 - Mac Graw-Hill - 1989. 
[25]- Oller S., Oñate E., Miquel J., and Botello S. - A Finite model for analysis of multiphase composite materials - Ninth International Conference on Composite materials - Madrid - Spain 1993.

[26]- Oller S., Oñate E. and Miquel J. - Simulation of anisotropic elastic-plastic behaviour of materials by means of an isotropic formulation - 2nd U.S. National Congress on Computational Mechanics - Washington - USA 1993. 